\title{
Vesicle cholesterol controls exocytotic fusion pore
}

\author{
Boštjan Rituper $^{\mathrm{a}}$, Alenka Guček ${ }^{\mathrm{a}, \S}$, Marjeta Lisjak ${ }^{\mathrm{a}}$, Urszula Gorska ${ }^{\mathrm{a}, \#}$, Aleksandra Šakanovićc \\ Saša Trkov Bobnar ${ }^{\mathrm{a}, \mathrm{b}}$, Eva Lasič ${ }^{\mathrm{a}}$, Mićo Božić ${ }^{\mathrm{a}}$, Prabhodh S. Abbineni ${ }^{\mathrm{d}}$, Jernej Jorgačevski ${ }^{\mathrm{a}, \mathrm{b}}$, \\ Marko Kreft ${ }^{\mathrm{a}, \mathrm{b}}$, Alexei Verkhratsky ${ }^{\mathrm{b}, \mathrm{e}, \mathrm{f}}$, Frances M. Platt ${ }^{\mathrm{g}}$, Gregor Anderluh ${ }^{\mathrm{c}}$, \\ Matjaž Stenovec $^{\mathrm{a}, \mathrm{b}}$, Bojan Božič ${ }^{8}$, Jens R. Coorssen ${ }^{9}$, Robert Zorec ${ }^{\mathrm{a}, \mathrm{b}, ¥, *, *}$

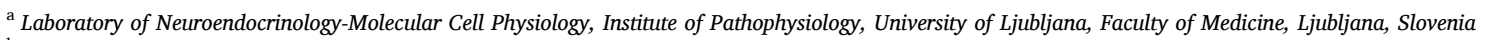 \\ ${ }^{\mathrm{b}}$ Celica Biomedical, 1000, Ljubljana, Slovenia \\ ${ }^{\mathrm{c}}$ Laboratory for Molecular Biology and Nanobiotechnology, National Institute of Chemistry, Ljubljana, Slovenia \\ d Department of Pharmacology, University of Michigan, Ann Arbor, MI 48109-5632, United States of America \\ ${ }^{\mathrm{e}}$ Faculty of Biology, Medicine and Health, The University of Manchester, Manchester, M13 9PT, United Kingdom \\ ${ }^{\mathrm{f}}$ Achucarro Center for Neuroscience, IKERBASQUE, 48011 Bilbao, Spain \\ ${ }^{g}$ Department of Pharmacology, University of Oxford, Oxford, OX1 3QT, United Kingdom \\ ${ }^{8}$ Institute of Biophysics, Faculty of Medicine, University of Ljubljana, Slovenia \\ ${ }^{9}$ Department of Health Sciences, Faculty of Applied Health Sciences and Department of Biological Sciences, Faculty of Mathematics \& Science, Brock University, St \\ Catherine's, Ontario, Canada
}

\section{A R T I C L E I N F O}

\section{Keywords:}

Cholesterol

Exocytosis

Constriction force

Fusion pore conductance

Fusion pore widening and constriction

Lysosomal storage disease

\begin{abstract}
A B S T R A C T
In some lysosomal storage diseases (LSD) cholesterol accumulates in vesicles. Whether increased vesicle cholesterol affects vesicle fusion with the plasmalemma, where the fusion pore, a channel between the vesicle lumen and the extracellular space, is formed, is unknown. Super-resolution microscopy revealed that after stimulation of exocytosis, pituitary lactotroph vesicles discharge cholesterol which transfers to the plasmalemma. Cholesterol depletion in lactotrophs and astrocytes, both exhibiting $\mathrm{Ca}^{2+}$-dependent exocytosis regulated by distinct $\mathrm{Ca}^{2+}$ sources, evokes vesicle secretion. Although this treatment enhanced cytosolic levels of $\mathrm{Ca}^{2+}$ in lactotrophs but decreased it in astrocytes, this indicates that cholesterol may well directly define the fusion pore. In an attempt to explain this mechanism, a new model of cholesterol-dependent fusion pore regulation is proposed. High-resolution membrane capacitance measurements, used to monitor fusion pore conductance, a parameter related to fusion pore diameter, confirm that at resting conditions reducing cholesterol increases, while enrichment with cholesterol decreases the conductance of the fusion pore. In resting fibroblasts, lacking the Npc1 protein, a cellular model of LSD in which cholesterol accumulates in vesicles, the fusion pore conductance is smaller than in controls, showing that vesicle cholesterol controls fusion pore and is relevant for pathophysiology of LSD.
\end{abstract}

\section{Introduction}

Membrane fusion is fundamental to the life of eukaryotic cells. It contributes to diverse cellular processes, including the vesicle-based secretion [1], which is essential for cell-to-cell signaling. Vesicular secretion is affected in lysosomal storage diseases (LSD), a group of inherited diseases caused by the deficiency of lysosomal and non-lysosomal proteins with consequent lysosome accumulation of several types of substrates, including cholesterol [2, 3]. Regulated exocytosis, which proceeds through a hemi-fusion contact [4] to the merger between the vesicle membrane and the plasma membrane (PM), leads to the formation of a fusion pore, through which molecules stored in the vesicle lumen exit into the extracellular space [5]. The view that emerged decades ago [6], assumes that once a fusion pore forms, the

\footnotetext{
* Corresponding author at: Univerza v Ljubljani, Lab Neuroendo Inst Pathophysiol, 1000 Ljubljana, Slovenia.

E-mail address: robert.zorec@mf.uni-lj.si (R. Zorec).

$\S$ Present address: Department of Medical Cell Biology, Uppsala University, Uppsala, Sweden

\# Present address: Smoluchowski Institute of Physics, Jagiellonian University, Krakow, Poland

¥ Lead Contact
} 
vesicle membrane abruptly and fully merges with the PM (full exocytosis). However, recent studies revealed that open fusion pore may partially or fully close again after a while (transient or reversible exocytosis). Multiple stages that a vesicle undergoes during exocytosis were confirmed by recordings of discrete steps in membrane capacitance $\left(\mathrm{C}_{\mathrm{m}}\right)$, linearly related to the membrane area, and affected by vesicle fusion and fission [7].

Progression through the stages of vesicle-PM fusion depends on vesicle size [8-10]. In synaptic communication, specialized for fast signaling, vesicles with relatively small diameters predominantly undergo full exocytosis [11-13]. The fusion pore opening, triggered by rapid $\mathrm{Ca}^{2+}$ rise, appears to be mediated by the soluble N-ethylmaleimide-sensitive factor attachment protein receptors (SNAREs), responsible for membrane fusion [1].

The structure of the fusion pore wall is under debate, although it is widely considered to be proteolipidic in nature [5]. Therefore, it is likely that both membrane protein and lipid molecules affect the formation and properties of the fusion pore, and stabilizing its structure. We examined whether cholesterol, the most common steroid in humans and a major constituent of the cell membrane, affects fusion pore conductance that reports fusion pore diameter [5], by monitoring changes in $\mathrm{C}_{\mathrm{m}}$ due to single vesicle interaction with the PM [7, 14].

Previous studies indicated that cholesterol regulates exocytosis. Possible explanations for the role of cholesterol in fusion might include the segregation of membrane proteins into domains, including the SNARE proteins $[2,15,16]$, and cholesterol ability to order membrane lipids and fluidity $[17,18]$. Within the PM this may affect transfer of molecules, including cholesterol, between the membrane density-rich domains to regions with lower density, generating tension [19]. Cholesterol potentially affects negatively curved lipid structures that are thought to be intermediates in membrane fusion [20-28].

Studies in defined in-vitro systems revealed that cholesterol may increase the efficiency of full exocytosis [29, 30] as well as the open probability of flickering fusion pores [26, 31], without affecting the fusion pore size [31]. The cholesterol role in promoting secretion has been confirmed in some mammalian cell models, including neuroendocrine PC12 [32], chromaffin cells [23], platelets [33] and pituitary cells $[20,25]$, where cholesterol appears to be necessary for the fusion of vesicle and PMs. However, another study demonstrated that cholesterol facilitates spontaneous and inhibits evoked synaptic secretion [34]. In insulin-secreting cells, excess of cholesterol inhibits vesicle exocytosis [35]. These contradictory findings reflect multiple pathways regulated by cholesterol, perhaps recruiting different regulatory factors at various stages of vesicle interactions with the PM [16], for example by affecting the coupling between $\mathrm{Ca}^{2+}$ sources and vesicle release sites [36, 37]. We investigated how acute cholesterol depletion affects vesicle discharge and whether steady-state cholesterol depletion/enrichment changes fusion pore conductance in the absence of physiological stimulation. We used electrically excitable pituitary lactotrophs and nonexcitable astrocytes, which exhibit distinct $\mathrm{Ca}^{2+}$ sources regulating exocytosis [14, 38]. We also studied how excess vesicle cholesterol affects fusion pore conductance in a cell model of LSD.

Using a cholesterol marker [39], we found that during stimulated exocytosis, vesicle cholesterol mixes with the PM. Depleting cholesterol by acute exposure of electrically excitable and nonexcitable cells to methyl- $\beta$-cyclodextrin (M $\beta C D$ ) stimulated vesicle cargo release independently of cytosolic $\mathrm{Ca}^{2+}$, suggesting that cholesterol content may regulate the conductance of the fusion pore directly. High-resolution measurements of $C_{m}$ and fusion pore conductance $\left(G_{p}\right)$, which report the electrical geometry of the fusion pore [40, 41], confirmed our theoretical predictions based on cholesterol transfer-induced membrane tension [19], that cholesterol depletion increases, while cholesterol enrichment decreases the $G_{p}$. Consistent with this hypothesis, cholesterol-rich vesicles in fibroblasts, exhibiting $\mathrm{Ca}^{2+}$-regulated exocytosis [42], lacking the Niemann-Pick Type $\mathrm{C} 1$ protein ( $\mathrm{Npc1}$ ), a cellular model of the human Niemann-Pick Type C1 lysosomal storage disease, exhibited a lower $G_{p}$ than wild-type controls. Our findings provide a conceptual advancement of our understanding of membrane pores, showing that vesicle cholesterol regulates fusion pore conductance and is relevant to the pathophysiology of LSD.

\section{Methods}

\subsection{Materials and solutions}

Methyl- $\beta$-cyclodextrin (M $\beta C D$ ) and cholesterol were from SigmaAldrich. The cholesterol assay kit (Amplex Red) and Vybrant DiD celllabeling solution were from Invitrogen. All other chemicals were of the highest purity available. The extracellular (bath) solution (ECS) for lactotrophs contained $130 \mathrm{mM} \mathrm{NaCl}, 5 \mathrm{mM} \mathrm{KCl}, 8 \mathrm{mM} \mathrm{CaCl}_{2}, 10 \mathrm{mM} \mathrm{D}-$ glucose, $10 \mathrm{mM}$ HEPES (N-2-hydroxyethylpiperazine- $\mathrm{N}^{\prime}$-2-ethanesulfonic acid) at pH 7.2 (with $\mathrm{NaOH}$ ). The ECS for astrocytes contained $130 \mathrm{mM} \mathrm{NaCl}, 5 \mathrm{mM} \mathrm{KCl}, 2 \mathrm{mM} \mathrm{CaCl}_{2}, 1 \mathrm{mM} \mathrm{MgCl}_{2}, 10 \mathrm{mM}$ D-glucose, $10 \mathrm{mM}$ HEPES at pH 7.2 (with $\mathrm{NaOH}$ ). In experiments with $\mathrm{Ca}^{2+}$-deficient extracellular solution, $\mathrm{CaCl}_{2}$ was replaced with $\mathrm{NaCl}$. The osmolarity of the ECS was $\sim 300$ mOsm.

\subsection{Animals}

The experiments were performed on primary lactotroph cultures isolated from 30- to 60-day-old Wistar rats and primary astrocyte cultures isolated from 2- to 3-day old Wistar rats. The care of the experimental animals was in accordance with the International Guiding Principles for Biomedical Research Involving Animals developed by the Council for International Organizations of Medical Sciences and Directive on Conditions for Issue of License for Animal Experiments for Scientific Research Purposes (Official Gazette of the RS, nos. 40/85 and 22/ 87). The research conducted was approved by The Administration of the Republic of Slovenia for Food Safety, Veterinary and Plant Protection (Republic of Slovenia, Ministry of Agriculture, Forestry and Food, Dunajska cesta 22, 1000 Ljubljana), document no. U34401-47/2014/7.

\subsection{Cell cultures}

Lactotroph primary cultures were prepared as described [43]. After isolation, lactotrophs were plated onto poly-L-lysine $(1 \%, \mathrm{w} / \mathrm{v})$-coated coverslips and kept in high-glucose Dulbecco's modified Eagle's medium (Invitrogen) supplemented with $10 \%$ newborn calf serum and L-glutamine at $37{ }^{\circ} \mathrm{C}$ with $95 \%$ humidity and $5 \% \mathrm{CO}_{2}$. Primary cortical astrocyte cultures were prepared from the cerebral cortices of neonatal rats as described [44]. Cells were grown in Dulbecco's modified Eagle's medium supplemented with $10 \%$ fetal bovine serum, $1 \mathrm{mM}$ sodium pyruvate, $2 \mathrm{mM}$ L-glutamine, and $5 \mathrm{U} / \mathrm{mL}$ penicillin / $5 \mu \mathrm{g} / \mathrm{mL}$ streptomycin at $37{ }^{\circ} \mathrm{C}$ with $95 \%$ humidity and $5 \% \mathrm{CO}_{2}$. When cells reached confluence, they were shaken overnight, trypsinized, and plated into 2 $\mathrm{mL}$ cell culture tubes. After reaching confluence again, they were subcultured onto $22 \mathrm{~mm}$ poly-L-lysine-coated $(0.1 \%$, w/v) coverslips. Experiments were performed on lactotrophs and astrocytes within 3 days after plating.

Fibroblasts were prepared from Npc1 null mice. Briefly, BALB/cNctr-

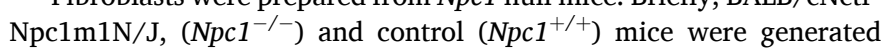
from heterozygous breeding. Genotyping was performed according to published methods [45]. All mice were maintained under a standard 12 $\mathrm{h}$ light/12 $\mathrm{h}$ dark cycle with water and food available ad libitum. All procedures were performed according to the UK Animals (Scientific Procedures) Act 1986 under a project license (PPL No. I25BD461E) from the UK Home Office. Fibroblasts were prepared from $\mathrm{Npc1}^{-/-}$and $N p c 1^{+/+}$mice according to published methods [46].

\subsection{Fluorescent (TopFluor) cholesterol loading}

Fluorescent cholesterol (TopFluor cholesterol)-M $\beta C D$ inclusion 
complexes were prepared as described [25] . Briefly, $10 \mathrm{mg}$ of TopFluor cholesterol (23-(dipyrromethene boron difluoride)-24-norcholesterol) (Avanti Polar Lipids) was added to the $10 \mathrm{mM} \mathrm{M} \beta \mathrm{CD}$ solution (in phosphate buffered saline [PBS], molar ratio of $\mathrm{M} \beta \mathrm{CD} /$ cholesterol 8:1) and sonicated in an ice bath $\left(4{ }^{\circ} \mathrm{C}\right)$ for $30 \mathrm{~min}$ protected from light. The solution was filtered through $0.2 \mu \mathrm{m}$ filters, aliquoted, and stored at $-20{ }^{\circ} \mathrm{C}$ until further use. To load fluorescent cholesterol into prolactin (PRL) secretory vesicles, lactotroph-loaded coverslips were washed with PBS and incubated with $1 \mathrm{~mL}$ of loading solution, diluted to a TopFluor cholesterol concentration of $50 \mu \mathrm{g} / \mathrm{mL}$ at $37{ }^{\circ} \mathrm{C}$ and $5 \% \mathrm{CO}_{2}$ for $60 \mathrm{~min}$. After thorough washing (in PBS), loading solution was replaced with standard lactotroph culture medium for $8-10 \mathrm{~h}$. High background fluorescence could be diminished by a brief ( $2 \mathrm{~min}$ ) incubation of cells with $10 \mathrm{mM} M \beta C D$ solution.

\subsection{Immunocytochemistry}

PRL granules attached to the cell surface were immunolabeled by first blocking nonspecific immunoreactive sites with $3 \%$ bovine serum albumin (BSA/PBS) and then tagging the PRL antigens with primary mouse monoclonal anti-PRL antibodies (1:300; Thermo Scientific) for $10 \mathrm{~min}$ at room temperature (RT). To immunolabel PRL secretory vesicles, cells were fixed and permeabilized with $2 \%$ formaldehyde (10 min, RT) and incubated in $3 \%$ BSA and $10 \%$ goat serum in PBS $\left(1 \mathrm{~h}, 37^{\circ} \mathrm{C}\right)$. PRL vesicles were labeled with primary mouse monoclonal anti-PRL antibodies (1:300, Thermo Scientific) for $2 \mathrm{~h}$ at $37^{\circ} \mathrm{C}$. Primary antiPRL antibodies were localized using secondary anti-mouse antibodies conjugated to specified Alexa fluorophores (1:600, $45 \mathrm{~min}, 37{ }^{\circ} \mathrm{C}$ ). Glutamatergic and ATP-containing vesicles were immunolabeled by fixing astrocytes with $4 \%$ formaldehyde ( $15 \mathrm{~min}, \mathrm{RT}$ ). Astrocytes were then incubated in $3 \%$ BSA and $10 \%$ goat serum in PBS $\left(1 \mathrm{~h}, 37^{\circ} \mathrm{C}\right)$. Next, primary mouse monoclonal anti-VGLUT1 antibodies (1:800; SySy) were added for $2 \mathrm{~h}$ at $37{ }^{\circ} \mathrm{C}$ and then primary rabbit polyclonal anti-VNUT antibodies (1:200, from Prof. Yoshinori Moriyama) for $2 \mathrm{~h}$ at $37{ }^{\circ} \mathrm{C}$, followed by the addition of both secondary antibodies conjugated to specified Alexa fluorophores (1:600, $\left.45 \mathrm{~min}, 37^{\circ} \mathrm{C}\right)$. SlowFade Gold antifade reagent was used to mount coverslips to glass slides (Invitrogen).

\subsection{EGFP-D4-PFO- and mCherry-D4-PFO expression and purification}

The perfringolysin O (PFO) D4 domain with N-terminal EGFP (EGFPD4-PFO) or mCherry (mCherry-D4-PFO) fusion was expressed and purified as described previously [39]. Briefly, an EGFP- or mCherry-tagged D4 DNA fragment coding for the amino acids from S386 to N500 was introduced into the pET8c vector using XhoI and MluI sites. The resulting expression plasmid encoded for $\mathrm{N}$-terminal $6 \mathrm{xHis}$-tag followed by the recognition site for TEV protease and the EGFP/mCherry coding region separated from the D4 PFO domain with 10 amino acids long GS linker. The integrity of each plasmid was verified by DNA sequencing of its entire open reading frame. For recombinant protein production, a freshly transformed Escherichia coli BL21(DE3) strain was used, which was grown at $37{ }^{\circ} \mathrm{C}$ in $\mathrm{LB}$ medium supplemented with $100 \mu \mathrm{g} / \mathrm{mL}$ ampicillin. Overexpression of the fluorescently tagged D4 domain was induced with $0.5 \mathrm{mM}$ isopropyl $\beta$-D-1-thiogalactopyranoside (IPTG) at $\mathrm{OD}_{600}$ from 0.5 to 0.7 for $16-18 \mathrm{~h}$ at $20^{\circ} \mathrm{C}$. The cells were harvested by centrifugation, resuspended in $10 \mathrm{~mL} / \mathrm{g}$ wet mass of lysis buffer $(50 \mathrm{mM}$ $\mathrm{NaH}_{2} \mathrm{PO}$, $300 \mathrm{mM} \mathrm{NaCl}, 10 \mathrm{mM}$ imidazol [pH 8.0]) and lysed by sonication. Recombinant proteins were purified by nickel chromatography (Ni-NTA Superflow; Qiagen) using the same procedures as described previously [39]. Purified protein was dialyzed against Tris-HCl buffer (50 mM Tris- $\mathrm{HCl}, 200 \mathrm{mM} \mathrm{NaCl}, 5 \%$ (v/v) glycerol [pH 7.4]) using Slide-a-lyzer with a molecular weight cutoff of $10 \mathrm{kDa}$ (Thermo Scientific). Purification was confirmed by SDS-PAGE and Coomassie staining.

\subsection{D4-PFO membrane and organelle labeling, and D4-PFO expression in mammalian cells}

To label the exoplasmic leaflet of lactotroph PM, cells were first rinsed with PBS followed by incubation with $1 \mu \mathrm{M}$ mCherry-D4-PFO or EGFP-D4-PFO in 3\% BSA for $30 \mathrm{~min}$ at RT. After washing, cells were fixed with $2 \%$ formaldehyde. In some experiments, cells were further immunolabeled as described in the Results (Fig. 3). In addition to the labeling, the mCherry-D4-PFO-encoding plasmid was also expressed in lactotrophs. For this purpose, gene fragment coding for mCherry-D4 fusion from the bacterial expression plasmid described above was amplified with the following pair of primers: $5^{\prime}$-CGT ACA AGC TTA TGC ATC ACC ATC ACC ATC AC-3' (sense) and 5'-GCA TGT GGA TCC AGT ACG CGT TTA ATT GTA AGT AAT ACT-3' (antisense). The PCR product was cloned into the pcDNA3 vector using HindIII and BamHI restriction sites. The sequence of the constructed plasmid was confirmed by DNA sequencing. Electroporation was used to transfect plasmids into lactotrophs using a Basic Nucleofector Kit for Primary Mammalian Epithelial Cells (Lonza, program W-001) (Figure S1).

To examine the distribution of cholesterol-rich membranous organelles in fibroblasts (wild-type and $\mathrm{NPC}^{-/-}$lacking the vesicle cholesterol transporter Niemann-Pick Type C1), cells were washed (3 min) with PBS, fixed in formaldehyde (2\% in PBS) for $10 \mathrm{~min}$, washed three times ( $3 \mathrm{~min}$ ) with PBS, exposed to D4-PFO-mCherry $(0.25 \mu \mathrm{M})$ for 30 $\mathrm{min}$, and washed three times with PBS ( $3 \mathrm{~min}$ ); all at RT. Finally, coverslips were mounted onto glass slides using SlowFade Gold antifade mountant (Thermo Scientific).

\subsection{Plasma membrane labeling with Vybrant DiD solution}

In some experiments, plasma membrane was labeled with $25 \mu \mathrm{M}$ Vybrant DiD cell-labeling solution (Invitrogen) for $10 \mathrm{~min}$ at RT. Cells were then fixed in $2 \%$ formaldehyde and mounted on glass slides using SlowFade Gold antifade reagent (Invitrogen).

\subsection{Electrophysiological recordings}

Cell-attached capacitance measurements were performed with a dual-phase lock-in patch-clamp amplifier (SWAM IIC; Celica) as described [43]. All experiments were performed at RT. Individual recordings lasted $1000 \mathrm{~s}$. We used fire-polished pipettes, heavily coated with Sylgard and with a tip resistance of 2-5 M $\Omega$. The bath and pipettes contained ECS. A sine wave $(111 \mathrm{mV}$ rms and $1591 \mathrm{~Hz}$ for lactotrophs and $111 \mathrm{mV}$ rms and $6364 \mathrm{~Hz}$ for astrocytes) was applied to the pipette, and the pipette potential was held at $0 \mathrm{mV}$. During the experiments, the phase angle was adjusted to nullify the changes in the real $(R e)$ trace in response to the manually generated $10 \mathrm{fF}$ calibration pulses. Vesicle capacitance $\left(\mathrm{C}_{\mathrm{v}}\right)$ and fusion pore conductance $\left(\mathrm{G}_{\mathrm{p}}\right)$ were calculated from the imaginary $(\mathrm{Im})$ and real $(R e)$ parts of the admittance traces as reported previously [47]: $C_{v}=\left[\left(\operatorname{Re}^{2}+\operatorname{Im}^{2}\right) / \mathrm{Im}\right] / \omega$, where $\omega$ is the angular frequency ( $\omega=2 \pi f$, $f$ is the sine wave frequency) and $G_{p}=\left(\operatorname{Re}^{2}+\right.$ $\left.\mathrm{Im}^{2}\right) / R e$. The fusion pore radius was estimated using the equation $\mathrm{G}_{\mathrm{p}}=$ $\left(\pi R_{\text {pore }}{ }^{2}\right) /(\rho \lambda)$, where $R_{\text {pore }}$ represents the fusion pore radius, $\rho$ is the estimated resistivity of saline $(100 \Omega \mathrm{cm})$, and $\lambda$ the estimated length of a gap junction channel (15 nm) [48]. The vesicle diameter was calculated using a specific membrane capacitance $\left(\mathrm{C}_{\mathrm{sm}}\right)$ of $8 \mathrm{fF} / \mu \mathrm{m}^{2}$ (lactotrophs) or $10 \mathrm{fF} / \mu \mathrm{m}^{2}$ (astrocytes) as described [43]. We used a custom-written MATLAB (Math Works) subroutine (CellAn; Celica) to analyze fusion events.

\subsection{Total cell cholesterol measurements}

The total cell cholesterol (with cholesterol esters) was determined using the Amplex Red Cholesterol Assay Kit (Invitrogen). Cells were isolated as described above, however, no serum or serum substitutes were used. Samples were treated with $10 \mathrm{mM} \mathrm{M} \beta \mathrm{CD}$ for $30 \mathrm{~min}$ at $37^{\circ} \mathrm{C}$ 
in a $5 \% \mathrm{CO}_{2}$ atmosphere. For cholesterol replenishment, cells were washed and resuspended in $10 \mathrm{mM}$ cholesterol loading solution (M $\beta \mathrm{CD}$ / cholesterol molar ratio of 8:1), prepared as described previously [49] for an additional $30 \mathrm{~min}$. Subsequently, cells were lysed with CelLytic (Sigma-Aldrich), mixed with Amplex Red reagent, and incubated for 60 min at $37{ }^{\circ} \mathrm{C}$ in a $5 \% \mathrm{CO}_{2}$ atmosphere. The resulting fluorescence was measured using an EnSpire 2300 Multimode Plate Reader (PerkinElmer) with excitation at $560 \mathrm{~nm}$ and fluorescence emission detected at $590 \mathrm{~nm}$.

\subsection{Prolactin release measurements}

To measure PRL release, lactotrophs were densely plated on $22 \mathrm{~mm}$ coverslips and treated with vehicle or $10 \mathrm{mM} \mathrm{M} \beta C D$ for $30 \mathrm{~min}$. Then, the bath solution was collected, and the rat PRL enzyme immunoassay (ELISA) was performed according to the manufacturer's instructions (A05101, SPI bio; Bertin Pharma).

\subsection{ANP.emd release measurements}

Astrocytes were transfected with the plasmid encoding atrial natriuretic peptide tagged with emerald green fluorescent protein (ANP.emd; a gift from Dr. Ed Levitan, University of Pittsburgh, Pittsburgh, PA, USA) using a Rat Astrocyte Nucleofector Kit (Lonza). Transfected cells were resuspended in culture medium and subcultured onto poly-L-lysinecoated coverslips or 24-well plates (Nunc, Thermo Scientific) and maintained at $37{ }^{\circ} \mathrm{C}$ in an atmosphere of $5 \% \mathrm{CO}_{2}$ for $48-72 \mathrm{~h}$. The release of ANP.emd was estimated by measuring the fluorescence intensity of the bath solution at specified time points (EnSpire microplate reader; PerkinElmer) at the emission wavelength of $508 \mathrm{~nm}$. Fluorescence was excited at $488 \mathrm{~nm}$. Background fluorescence was subtracted, and the measured values were averaged and expressed as a relative increase in fluorescence, normalized to controls. A one-sample $t$ - test was used for statistical comparison.

\subsection{Calcium measurements}

Cells were loaded with $2 \mu \mathrm{M}$ Fluo-4 AM (Molecular Probes) for 30 min in ECS. After rinsing in ECS or $\mathrm{Ca}^{2+}$-free ECS for $15 \mathrm{~min}$, the cells were placed into the recording chamber and imaged with a confocal laser scanning microscope (Zeiss LSM 780). Measurements were performed with a Zeiss Plan-Apochromat oil immersion objective $(40 \times$ NA 1.3). An Ar-ion laser was used to excite Fluo-4 in combination with a 505-nm long-pass emission filter. Time-lapse images were acquired at a sampling rate of $1 \mathrm{~Hz}$. First, baseline fluorescence intensities were recorded for $3 \mathrm{~min}\left(\mathrm{~F}_{0}\right)$. Then, cells were treated with either vehicle, 10 $\mathrm{mM} M \beta C D$, or $10 \mathrm{mM}$ cholesterol loading solution, and fluorescent images were acquired for an additional $3 \mathrm{~min}$. To assess the effect of prolonged exposure to $\mathrm{M} \beta \mathrm{CD}$, fluorescence images were acquired for the initial $3 \mathrm{~min}$ and then for an additional $3 \mathrm{~min}$ after $30 \mathrm{~min}$ of incubation with M $\beta C D$. Fluo-4 fluorescence, which reports changes in $\left[\mathrm{Ca}^{2+}\right]_{\mathrm{i}}$, was measured in a region of interest covering the entire cell image. Resulting time-dependent fluorescence intensity traces were first normalized to the maximal fluorescence intensity in a given trace. The fluorescence intensity change was defined as $\Delta \mathrm{F} / \mathrm{F}_{0}=\left(\mathrm{F}(\mathrm{t})-\mathrm{F}_{0}\right) / \mathrm{F}_{0}$, expressed as a percentage. $F_{0}$ is the average non-stimulated fluorescence intensity of ten frames with the lowest relative intensity of fluorescence, and $\mathrm{F}(\mathrm{t})$ is the relative fluorescence intensity at a given time (Figure S4).

\subsection{Lipid extraction and HPTLC analysis}

Cells (astrocytes) were lysed with CelLytic M (Sigma-Aldrich) and lipids were extracted as described [49]. Lysed cells were suspended in $\mathrm{PBS}$, and $\mathrm{CH}_{3} \mathrm{OH}$ and $\mathrm{CHCl}_{3}$ were added sequentially at a ratio of 0.8:2:1 (PBS/ $\mathrm{CH}_{3} \mathrm{OH} / \mathrm{CHCl}_{3}, \mathrm{v} / \mathrm{v} / \mathrm{v}$ ), followed by vortexing and sonication for $30 \mathrm{~s}$. An aqueous solution of $1 \mathrm{M} \mathrm{NaCl}$ and $0.1 \mathrm{M} \mathrm{HCl}$ was added, followed by the addition of $\mathrm{CHCl}_{3}$ to reach a final ratio of 1.8:2:2
(PBS/ $\mathrm{CH}_{3} \mathrm{OH} / \mathrm{CHCl}_{3}, \mathrm{v} / \mathrm{v} / \mathrm{v}$ ). The sample was vortexed vigorously after each addition. The lower organic phase was dried under $\mathrm{N}_{2}$ and dissolved in $\mathrm{CHCl}_{3} / \mathrm{CH}_{3} \mathrm{OH}$ (2:1) for automated high-performance thin-layer chromatography (HPTLC) using the CAMAG AMD 2 system. Silica gel 60 plates (Merck) were pre-washed with $\mathrm{CH}_{3} \mathrm{OH} / \mathrm{C}_{4} \mathrm{H}_{8} \mathrm{O}_{2}(6: 4 \mathrm{v} / \mathrm{v})$, and heat activated at $110{ }^{\circ} \mathrm{C}$ for $30 \mathrm{~min}$ before analysis. Neutral lipids and phospholipids were resolved as previously described, detected using copper sulfate charring, and imaged using the LAS 4000 Biomolecular Imager (GE Healthcare) (Churchward et al., 2008a). Images were analyzed using Multi Gauge Software (FUJIFILM Corporation) or Image J (NIH). Fluorescence of control samples was defined as $100 \%$, and changes in lipid concentrations after treatment with $\mathrm{M} \beta C D$ are reported relative to corresponding controls.

\subsection{Microscopy and image analysis}

Except where stated otherwise, structured illumination microscopy (Zeiss Elyra PS1) was used to image fluorescently labeled cells. Images were acquired with a Zeiss Plan-neofluar oil immersion objective ( $63 \times$ NA 1.4). Cells were illuminated with laser lines of $488 \mathrm{~nm}$ and/or 561 $\mathrm{nm}$, and the emitted fluorescence was collected through band-pass emission filters (495-560 $\mathrm{nm}$ and 570-650 nm, respectively). Unless specified otherwise, $200 \mathrm{~nm}$-thick $\mathrm{Z}$ stacks were acquired with an EMCCD camera (16-bit, Andor iXon 885; Andor Technology) with variable exposure and analyzed with ImageJ.

\subsection{Analysis of D4-PFO-Labeled plasma membrane domains}

D4-PFO labeling and analysis protocols are depicted schematically in Figure S1. Briefly, after labeling with D4-PFO (and PRL in some experiments), single (D4-PFO) or dual channel (D4-PFO and PRL) fluorescent images were acquired and a $200 \mathrm{~nm}$ interval $\mathrm{Z}$ stack image was constructed for every cell. Then a 20-pixel-wide band covering the plasma membrane region was selected in every image within the stack (5-15 optical slices per cell), straightened, and thresholded automatically using the methods for D4-PFO [50] and PRL labeling [51]. Thresholded images were further analyzed as described in Fig. 3.

\subsection{Surface-Attached PRL cargo granules count}

Structured illumination microscopy images within the $\mathrm{Z}$ stack of lactotrophs (labeled for surface PRL granules; see Table S2) were recorded at $200 \mathrm{~nm}$ intervals, and the resulting $\mathrm{Z}$ stacks were used to reconstruct imaged cells in 3D. The 3D Objects Counter plugin [52] for ImageJ was used to calculate the number of PRL surface granules. Results were normalized to the cell volume, which was calculated as described [25].

\subsection{Estimation of secretory vesicle quantity}

Confocal laser scanning microscopy (Zeiss LSM 780) was used to image immunolabeled vesicles in fixed permeabilized lactotrophs and astrocytes. Images were acquired with a Zeiss Plan-Apochromat oil immersion objective (63 $\times$ NA 1.4). For excitation of Alexa Fluor 488, an Ar-ion laser was used in combination with a band-pass emission filter (505-530 nm), and for excitation of Alexa Fluor 543, a He-Ne laser was used in combination with a long-pass emission filter (cutoff below 560 $\mathrm{nm}$ ). Imaged cells were Z sectioned at 400-nm-thick intervals. Fluorescence images were thresholded automatically using an iterative selection method [53] for lactotrophs and another method [50] for astrocytes. The percentage cell area (in an optical slice) positive for immunolabeled vesicles was calculated by dividing above-threshold pixels with all the pixels in a region of interest covering the entire cell (cell borders were determined with the help of transmission images). 


\subsection{Analysis of D4-PFO-Labeled membranous organelles in fibroblasts}

To estimate the vesicle counts and vesicle intensity, confocal images were analyzed with ImageJ. The minimum fluorescent spot taken to identify an individual vesicle in a confocal image was three adjacent pixels $(0.132 \times 0.132 \mu \mathrm{m})$, and the minimum surface area covered by a spot was $0.052 \mu \mathrm{m}^{2}$. Considering that the minimum D4-PFO-positive vesicle consisted of three adjacent pixels, a broad span of vesicles with different sizes and intensity was covered by this analysis.
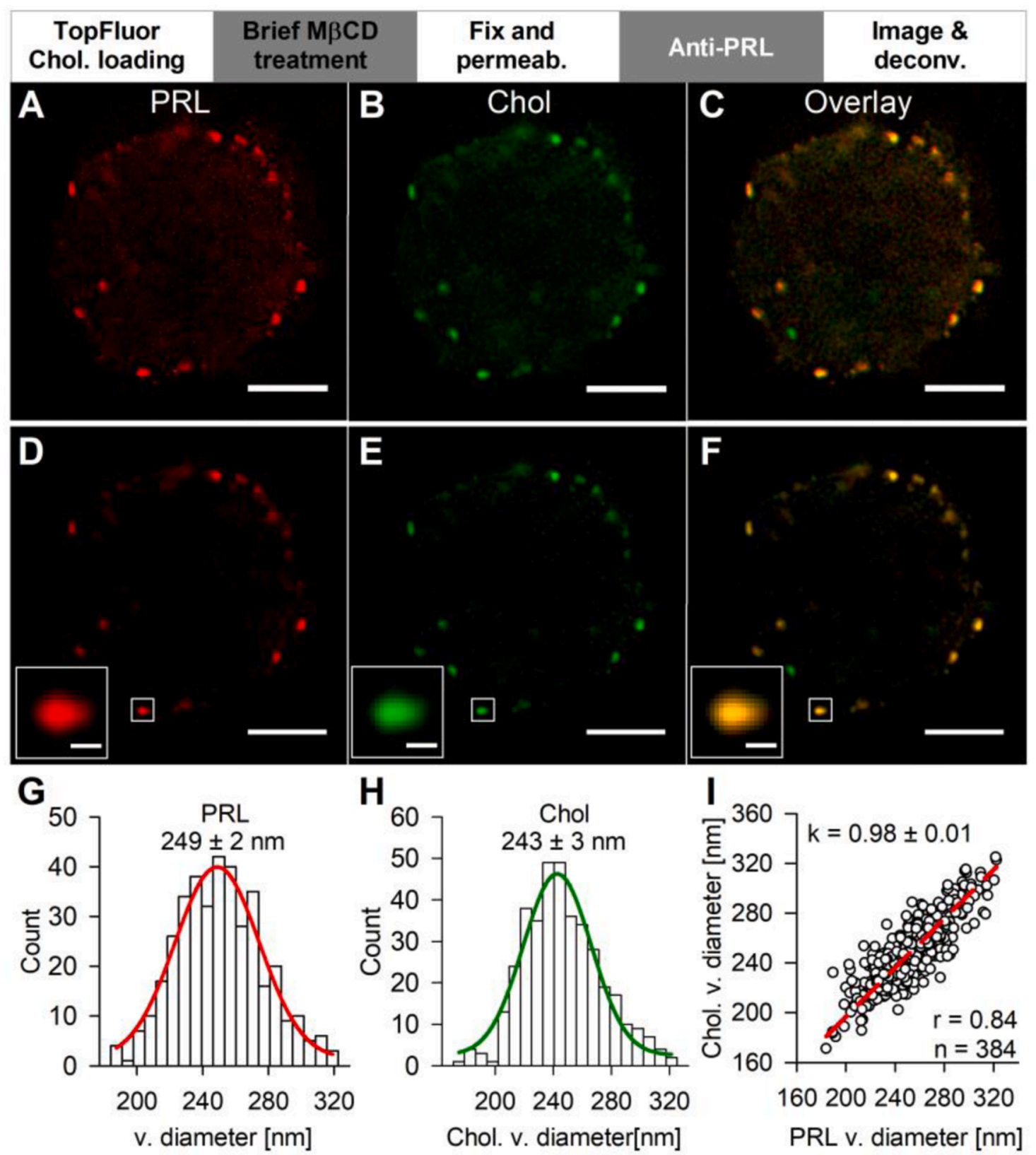

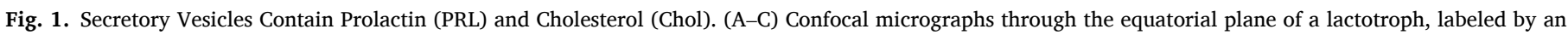
anti-PRL antibody (red, A) and fluorescent TopFluor-labeled cholesterol (Chol, green, B). Protocol is shown on the top of these panels, where the TopFluor labeling was followed by a brief $(2 \mathrm{~min})$ exposure of cells to methyl- $\beta$-cyclodextrin $(+\mathrm{M} \beta \mathrm{CD}, 10 \mathrm{mM})$, followed by permeabilization and fixation. Subsequently cells were exposed to anti-PRL antibodies to label vesicle content (see Methods). (C) PRL and Chol signals are overlaid (colocalized pixels in yellow). Note that in panel B, the plasma membrane is only faintly labelled due to the brief exposure of cells to MBCD as reported [25]. (D-F) Deconvoluted images from panels A to C, anti-PRL antibody staining (red, D) and fluorescent TopFluor-labeled cholesterol (green, E). (F) PRL and Chol signals are overlaid (colocalized pixels in yellow). Insets in the lower left corners (D-F) represent colocalized PRL and Chol in a vesicle.

(G) Frequency distribution (Count) of PRL vesicles versus their diameter (PRL v. diameter). The red line represents the fitted Gaussian (see also Figure S1) with a mean PRL vesicle diameter of $249 \pm 2 \mathrm{~nm}(n=384$; bin width $10 \mathrm{~nm}$; ten cells).

(H) Frequency distribution (Count) of Chol vesicles versus their diameter. The green line depicts the fitted Gaussian with a mean vesicle diameter of $243 \pm 3 \mathrm{~nm}(n=$ 384; bin width $10 \mathrm{~nm}$; ten cells).

(I) PRL vesicle (v.) diameter plotted versus Chol vesicle (v.) diameter. The red line was obtained by a linear regression (passing through the origin) of the form: Chol. v. diameter $=k \times$ PRL v. diameter; correlation coefficient, $r=0.84(p<0.001)$; the slope of the line $k=0.98 \pm 0.01$ (in red; $p<0.001)$. Scale bar, $3 \mu$ m; inset scale bar, $200 \mathrm{~nm}$ in (D-F). 


\subsection{Statistical tests}

Experimental data were compared with either the Student's $t$-test, one-way ANOVA with Holm-Sidak's post-hoc test or ANOVA on Ranks with Dunn's post-hoc test, as indicated. Depending on the distribution, the data are presented as means \pm SEM or medians with interquartile range. Differences were considered significant at $* p<0.05,{ }^{* *} p<0.01$, and $* * * p<0.001$. Statistical analyses were carried out using SigmaPlot (Systat Sofware). Data in Figure S4 were fitted using standard model linear regression, and the slopes were statistically compared with the analysis of covariance (ANCOVA) method.

\section{Results}

\subsection{Secretory vesicles contain cholesterol}

Lactotroph vesicles store and secrete prolactin (PRL). To determine whether PRL and cholesterol are stored in the same vesicles the cells were labeled with anti-PRL antibodies (Fig. 1A) together with fluorescent (TopFluor) cholesterol (Fig. 1B), which has similar properties to native cholesterol [54]. Exposure of cells to TopFluor for $60 \mathrm{~min}$ predominantly labeled the PM [25]. However, if such exposure lasted 8 $10 \mathrm{~h}$ and was followed with a short ( $2 \mathrm{~min}$ ) wash of cells with M $\beta C D$, the visualization of intracellular structures was improved (see Methods), thus revealing that most vesicles contain both markers (Fig. 1C). Using deconvolution, diameters of structures with both markers (Figs. 1D-1F) were determined, by constructing intensity profiles of labeled structures, fitting these profiles with Gaussian curves and measuring full width at half maximum $\left(\mathrm{FWHM}=2 \sigma(2 \ln 2)^{1 / 2}\right.$, where $\sigma$ denotes standard deviation) (Fig. 1F, inset). The PRL- and TopFluor-positive structures ( $n=$ 384, from ten cells) have similar diameter: $249 \pm 2 \mathrm{~nm}$ (Figs. 1G) and $243 \pm 3 \mathrm{~nm}$ (Fig. 1H), respectively. Fitting the PRL- and TopFluor-positive vesicle diameters by linear regression yielded a slope of $0.98 \pm 0.01$ (correlation coefficient $r=0.84, p<0.001, n=384$;
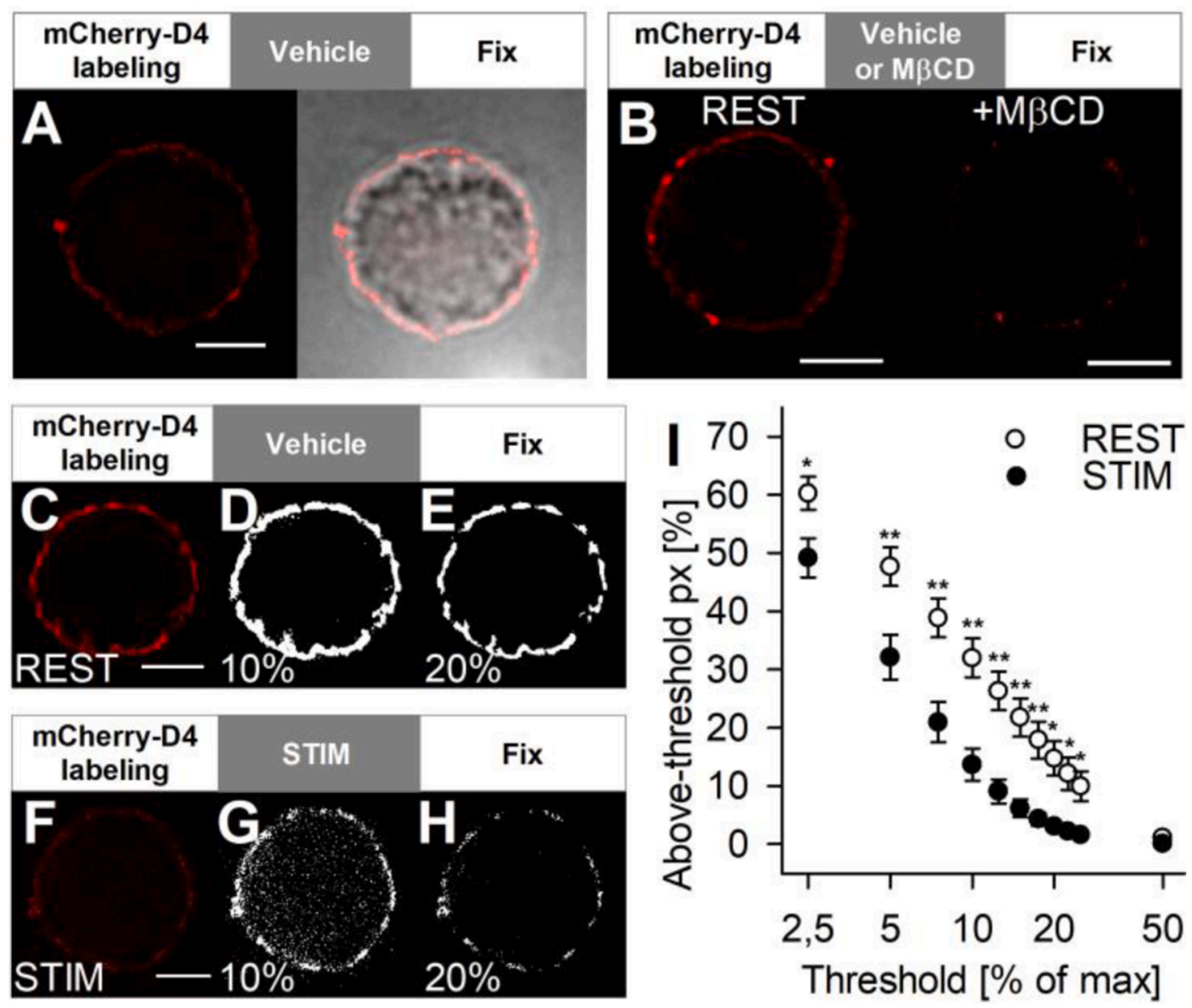

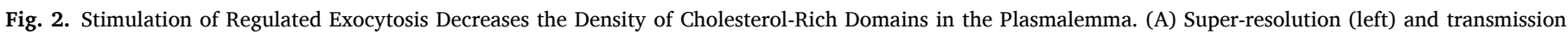

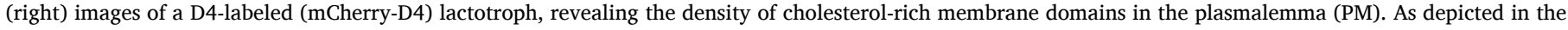

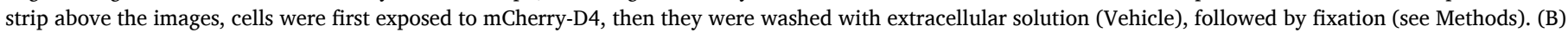

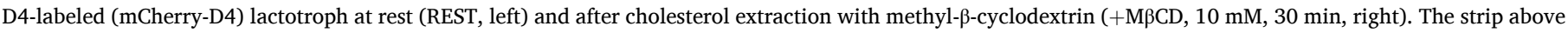

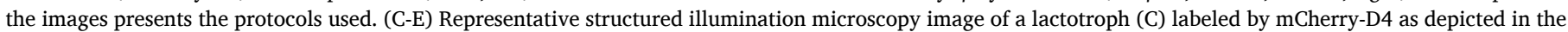

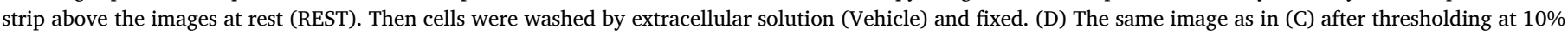

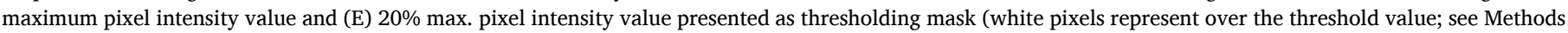

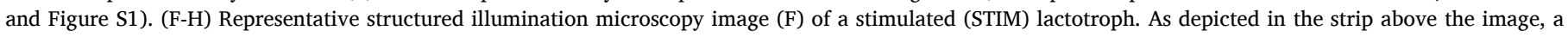

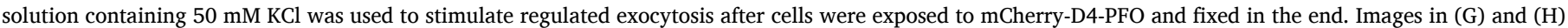

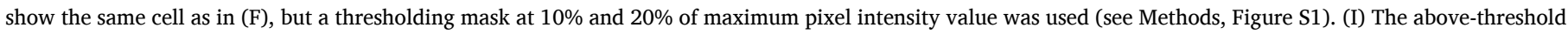

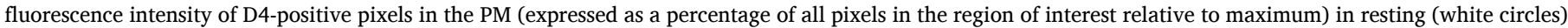

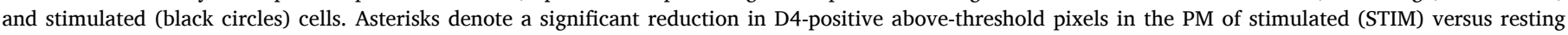

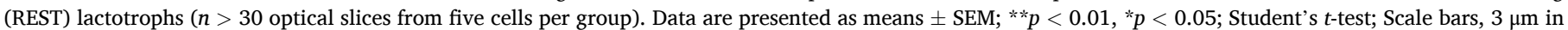
(A) $-(\mathrm{F})$. 
Fig. 1I), indicating that cholesterol may be present in PRL-containing secretory vesicles, consistent with earlier report [55]. Thus stimulation of regulated exocytosis may instigate the PM-directed transfer of vesicle cholesterol, thus affecting the PM cholesterol composition.

\subsection{Stimulation of exocytosis reduces cholesterol-rich pm domains}

To test how stimulated exocytosis affects cholesterol-rich PM domains, we first used a cholesterol-specific marker, mCherry-D4-PFO (D4) $[39,56,57]$, to visualize the cholesterol content in the PM [25]. Cells were exposed to D4, washed subsequently with extracellular solution (vehicle), and finally fixed with $2 \%$ formaldehyde (strip above Fig. 2A). The D4 labeled the PM (Fig. 2A). In M 3 CD-treated (10 mM, 30 min) cells [25, 27], the PM D4 staining was strongly reduced (Fig. 2B, right), indicating that D4 associates with the cholesterol-rich domains in the PM. The extent of D4 labeling was determined by delineating a band overlaying the PM (Figure S1) and calculating the percentage of D4-positive pixels, relative to all the pixels in the region of interest. $\mathrm{M} \beta \mathrm{CD}$ reduced D4 labeling from $9.0 \% \pm 0.5 \%$ in controls to $2.1 \% \pm$ $0.3 \%$ in M $\beta C D$-treated cells ( $p<0.001, n=50$ optical slices from 5 cells per group). This is consistent with the cholesterol content determined biochemically (Amplex Red, see Methods) in M $\beta C D$-treated pituitary cells, which was $56.8 \% \pm 10.6 \%$, normalized to controls. A similar reduction to $52.3 \% \pm 4.9 \%$ was also observed in astrocytes (Table S1). M $\beta C D$ selectively extracted cholesterol from cells without affecting other membrane lipids, as quantified by high-performance thin layer chromatography (Table S1).

Second, we tested whether the distribution of cholesterol in the PM was affected by regulated exocytosis. To trigger exocytosis D4-labeled cells were depolarized with $50 \mathrm{mM} \mathrm{KCl}$ extracellular solution [58] and were subsequently examined by microscopy. D4-labeling of the PM was reduced in the stimulated vs. resting cells (compare Figs. 2C-E with F-H). As the decrease in the signal depends on the noise level, we assessed the changes in D4 area at several intensity thresholds (see Figs. 2D, E and 2 G, H). The D4-labeled pixels above the threshold were significantly reduced in exocytosis-stimulated cells (Fig. 2I; $n=30$ optical slices, five cells), indicating that the D4-unlabeled cholesterol, delivered from vesicles, diluted the D4-labeled cholesterol in the PM. By readjusting the acquisition parameters for faint signals, we further tested the density reduction of D4-positive domains after stimulation of exocytosis $(p<$ 0.001 , Figures S2A and S2B), and also determined that the size of individual D4 domains (see Figs. 2D, E and $2 \mathrm{G}, \mathrm{H}$ ) decreased after stimulation of regulated exocytosis ( $p<0.05$, Figures S2C and S2D).

These data indicate that stimulation of regulated exocytosis changes cholesterol distribution in the PM.

\subsection{Vesicle cholesterol transfer to the PM and prolactin release}

To further examine whether regulated exocytosis initiates cholesterol redistribution to the PM, we examined whether proximity between the PM-located D4 is related to the PRL vesicles and exocytosed PRL aggregates $[59,60]$ in resting and stimulated cells. Assuming regulated exocytosis promotes cholesterol delivery to the PM, the association (distance) between the D4- and PRL-labeled PM structures should change.

First, to determine the association between D4- and PRL-labeled structures at the PM, non-stimulated cells were exposed to D4. After washing with extracellular solution (Vehicle) and fixation, vesiclestored PRL within cells was labeled by anti-PRL antibodies (Fig. 3A). Next, to see whether the association of respective labels in stimulated cells is changed, the D4-labeling was followed by stimulation $(50 \mathrm{mM}$ $\mathrm{KCl}$, with and without D4) and then the surface-deposited PRL aggregates [61] were labeled by anti-PRL antibodies (Fig. 3B and 3C).

Types of associations between PRL- and D4-positive structures at the PM are shown in insets (Fig. 3C; arrows) which demonstrate that some structures are overlapping or in contact (i.e., are associated) and others are separated (not associated). The distance between the non-associated structures was quantified (Figures S1C and S1D) by labeling cells with mCherry-D4 and the anti-PRL-antibodies in resting (REST) and stimulated (STIM) conditions (Fig. 3A-C). This analysis revealed that stimulation-induced exocytosis significantly increased the D4-PRL distance (Fig. 3D) from $0.28 \pm 0.03 \mu \mathrm{m}$ (REST, $n=182$; nine cells) to 0.75 $\pm 0.13 \mu \mathrm{m}$ (STIM, $n=96$; ten cells, $p<0.001$; see also Figure S1). Stimulation of exocytosis also significantly decreased the D4-PRL associations, as the overlap of D4- and PRL-positive structures at the PM decreased (Fig. 3E) from $54.9 \% \pm 2.0 \%$ in controls (REST, $n=9$ cells) to $39.6 \% \pm 2.5 \%$ in exocytosis-stimulated cells (STIM, $n=24$ cells, $p<$ 0.01). The decrease in the D4-PRL association in both experiments arguably reflects delivery of unlabeled vesicle-stored cholesterol to the PM during stimulated exocytosis.

To directly visualize the exocytosis-delivered cholesterol, we included D4 into the stimulation solution (Fig. 3C). The degree of D4PRL association was significantly higher in this group of cells (Fig. 3E, red column, STIM + D4, $61.1 \% \pm 3.2 \%, n=16$ cells) versus STIM $(39.6 \% \pm 2.5 \%, n=24$ cells, $p<0.01)$. Moreover, the D4-positive PM area (relative to the total PM area) was also significantly higher in the STIM + D4 group (Fig. 3F; 9.9\% $\pm 0.5 \%, n=16$ cells) versus the STIM group $(7.7 \% \pm 1.0 \%, n=14$ cells, $p<0.05)$.

The delivery of vesicle cholesterol to the PM was tested by using a secondary labeling with the green D4 (EGFP-D4-PFO, EGFP-D4; Fig. 3G). The cholesterol-rich domains were first exposed to D4 (red fluorescence) to saturate the cholesterol-rich sites. Subsequently, we used a green fluorescent peptide, EGFP-D4, to visualize the newly added cholesterol after washing D4 with vehicle (controls, REST; Fig. 3H top panel) or stimulating exocytosis with $50 \mathrm{mM} \mathrm{KCl} \mathrm{(STIM,} \mathrm{Fig.} \mathrm{3H,} \mathrm{bottom}$ panel). As shown on Fig. 3I, the D4-positive pre-labeled (red) membrane area decreased significantly from $7.0 \% \pm 0.5 \%$ in controls (REST, red bar, $n=20$ cells) to $3.8 \% \pm 1.6 \%$ in exocytosis-stimulated cells (STIM, red bar, $n=19$ cells, $p<0.001$ ). However, the green fluorescent EGFPD4 PM area increased significantly from $1.2 \% \pm 0.1 \%$ in controls (REST, green bar, $n=20$ cells) to $1.6 \% \pm 0.1 \%$ in stimulated cells (STIM, green bar, $n=19$ cells, $p<0.01$; Fig. 3I).

These data are consistent with the view that vesicle cholesterol is delivered to the PM in the process of exocytosis.

\subsection{Exocytotic release stimulated by cholesterol extraction}

To address the controversies regarding the effect of cholesterol extraction on exocytotic release [20, 25, 35], we exposed cells to $10 \mathrm{mM}$ $\mathrm{M} \beta \mathrm{CD}$ for $30 \mathrm{~min}$. This reduced membrane cholesterol levels (Fig. 2B) without affecting other membrane lipids (Table S1), consistent with previous reports $[20,21]$. Next, we probed whether cholesterol extraction with M $\beta C D$ affects exocytotic release in electrically excitable lactotrophs and electrically non-excitable astrocytes.

Application of $M \beta C D$ to lactotrophs significantly increased the cumulative release of PRL over a period of $30 \mathrm{~min}$ (Fig. 4A) from $0.54 \pm$ $0.15 \mathrm{ng} / \mathrm{mL}$ in controls (REST) to $2.48 \pm 0.10 \mathrm{ng} / \mathrm{mL}$ in treated cells ( $+\mathrm{M} \beta \mathrm{CD}, p<0.01 ; n=4$ ELISA reactions). In astrocytes expressing ANP. emd peptide, which accumulates in secretory vesicles and is released by regulated exocytosis [62], $\mathrm{M} \beta \mathrm{CD}$ treatment $(10 \mathrm{mM}, 30 \mathrm{~min})$ increased secretion of ANP.emd (Fig. 4B), measured as changes in the bath fluorescence relative to the control. Assuming a linear relationship between the bath fluorescence intensity change and ANP.emd release, M $\beta C D$ treatment triggered on average $26 \%$ more ANP.emd release compared with controls. M $\beta C D$ treatment did not cause any significant cell death within the relevant time interval, indicating that cell lysis did not contribute to an increase in signal fluorescence.

To validate these results at the single-cell level, we first imaged secreted PRL-containing aggregates on the cell surface of lactotrophs [61] and the non-secreted, cytoplasmic, PRL-containing vesicles (Figs. 4C-F). During 30-min exposure to $\mathrm{M} \beta \mathrm{CD}$, the normalized number of PM surface-attached PRL aggregates significantly increased (Fig. 4D) 

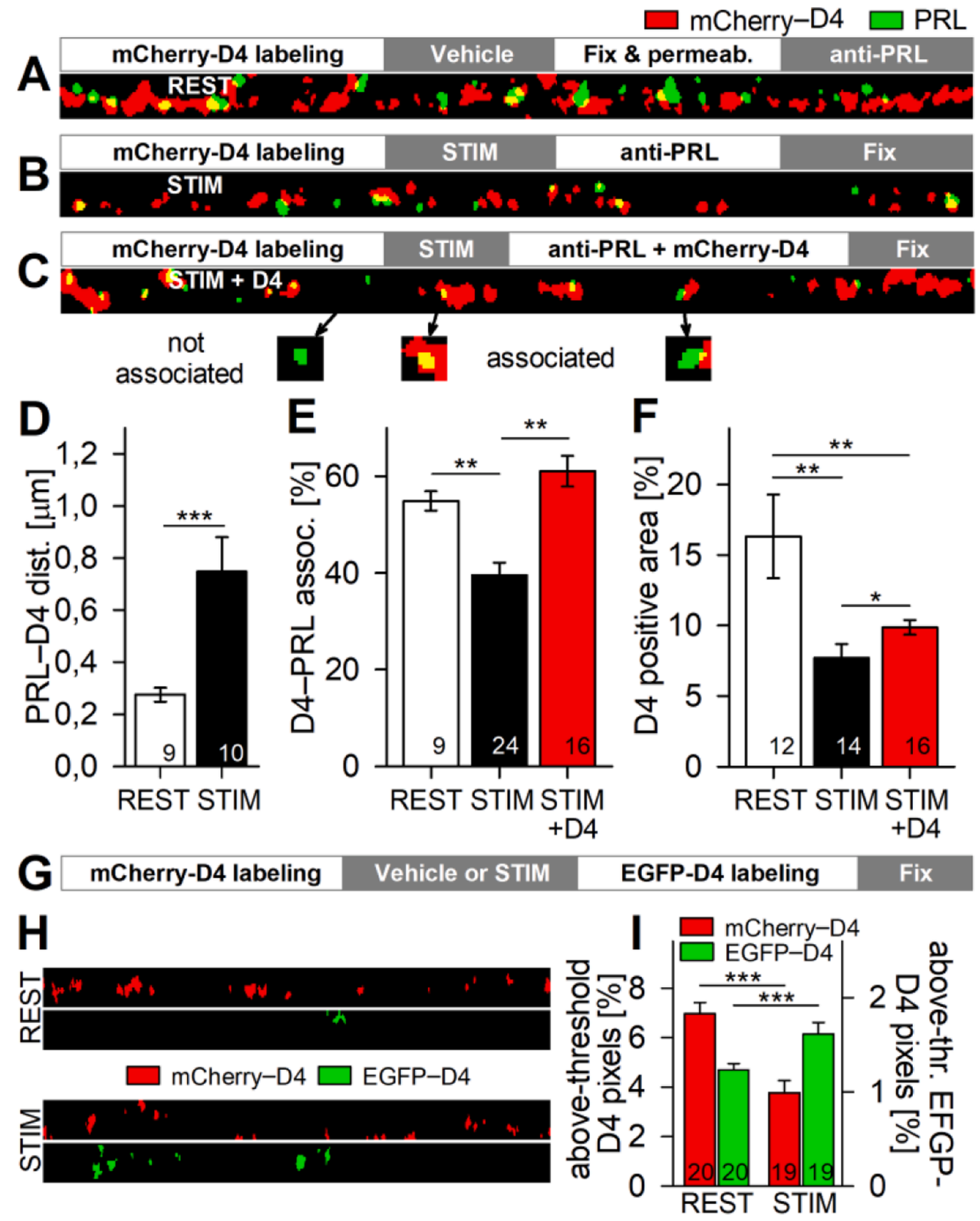


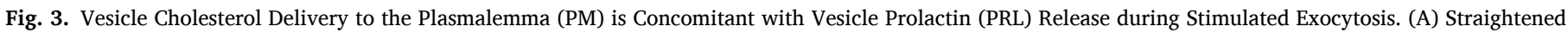

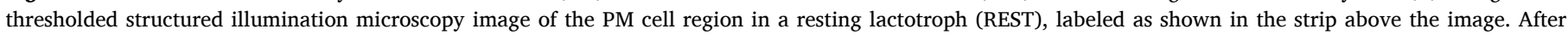

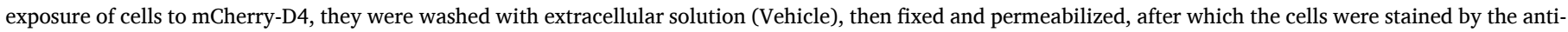

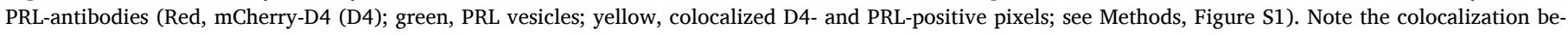

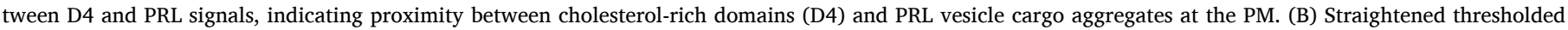

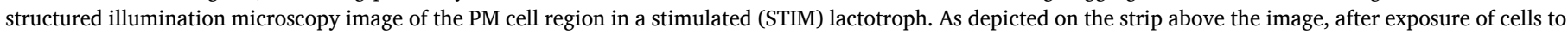

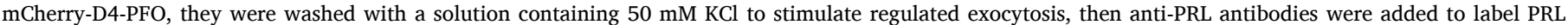

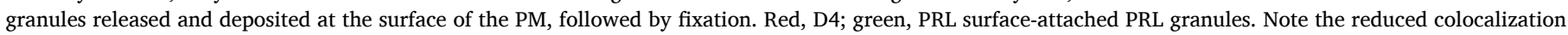

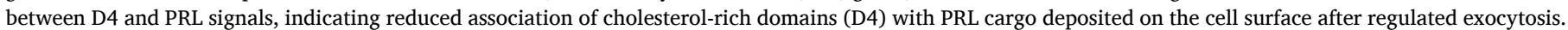

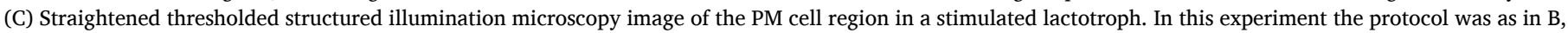

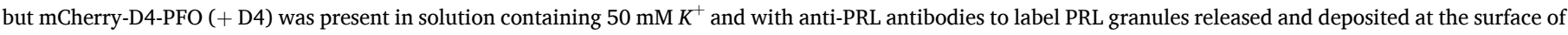

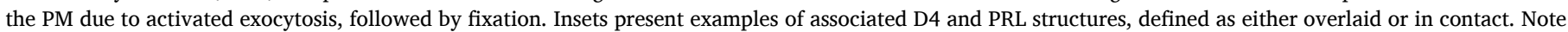

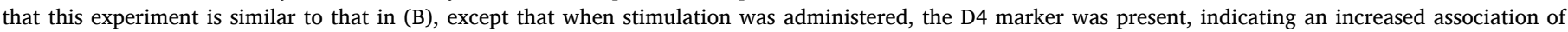

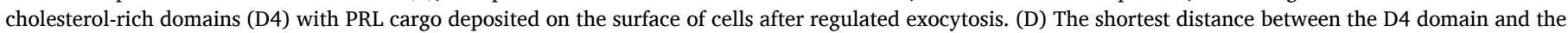

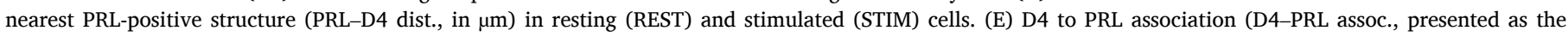

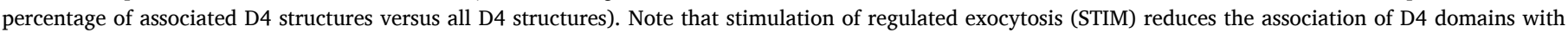

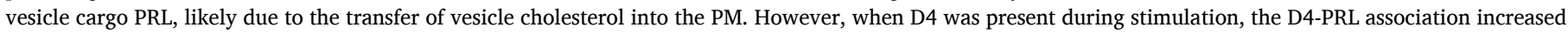

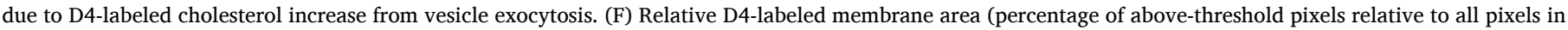

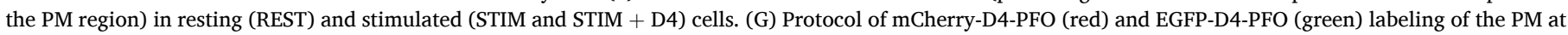

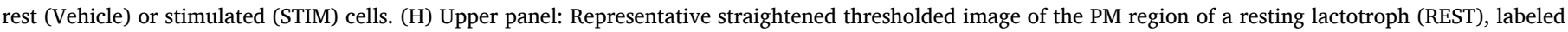

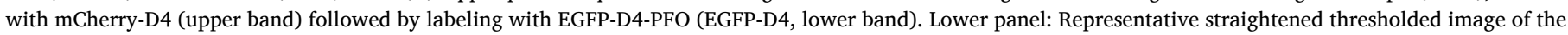

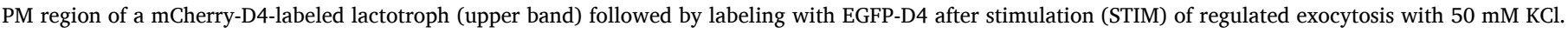

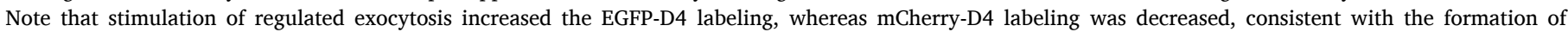

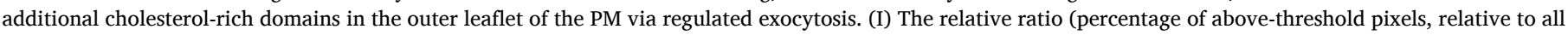

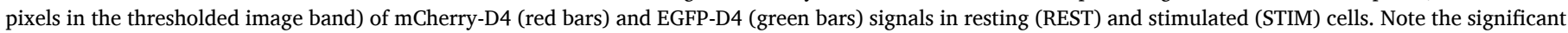

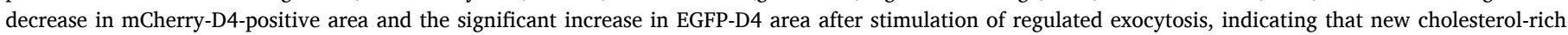

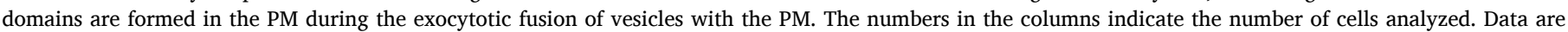
represented as means \pm SEM. ${ }^{* * *} p<0.001,{ }^{* *} p<0.01,{ }^{*} p<0.05$. Student's $t$-test in (D) and (I); ANOVA with Holm-Sidak post-hoc test in (E) and (F).

from $0.016 \pm 0.003$ aggregates $/ \mu \mathrm{m}^{3}$ in controls (REST, $n=13$ cells) to $0.103 \pm 0.016$ aggregates $/ \mu \mathrm{m}^{3}$ in treated cells ( $+\mathrm{M} \beta \mathrm{CD}, n=16$ cells, $p$ $<0.01$ ). Similarly, exposure to $\mathrm{M} \beta C D$ decreased the quantity of cytoplasmic PRL-loaded vesicles in lactotrophs, indicating a substantial stimulation of vesicle cargo discharge during cholesterol extraction (Fig. 4F). The surface area of above-threshold pixels (denoting cytoplasmic PRL vesicles; Fig. 4G), relative to all pixels in the cell, significantly decreased (Fig. $4 \mathrm{H}$ ) from $10.4 \% \pm 0.9 \%$ in controls (REST, $n=16$ cells) to $3.6 \% \pm 1.0 \%$ in treated cells $(+\mathrm{M} \beta \mathrm{CD}, n=16$ cells, $p<0.001)$.

Treatment of astrocytes with M $\beta C D$ did not significantly change the above-threshold vesicle glutamate transporter 1 (VGLUT1) immunoreactivity that represents glutamate-containing vesicles [63] (Fig. 4I, 4K and $4 \mathrm{~L}$, top graph), nor the above-threshold vesicular nucleotide transporter (VNUT) signal that represents ATP-containing vesicles [64] (Fig. 4J, 4K and 4L, top graph), However, we observed significantly less VNUT- and VGLUT1-positive vesicles in the 2 - $\mu \mathrm{m}$-thick band region, adjacent to the PM (Fig. $4 \mathrm{~L}$, bottom graph -ii). In this region, M $\beta C D$ treatment significantly decreased the level of above-threshold VNUT-positive pixels from $0.38 \% \pm 0.05 \%$ in controls (VNUT, REST, $n=19$ cells) to $0.21 \% \pm 0.04 \%$ in treated cells (VNUT, $+\mathrm{M} \beta C D, n=23$ cells, $p$ $<0.01)$ as well as the amount of above-threshold VGLUT1-positive pixels from $0.17 \% \pm 0.02 \%$ in controls (VGLUT1, REST, $n=19$ cells) to $0.11 \% \pm 0.01 \%$ in treated cells (VGLUT1, + M $\beta$ CD, $n=23$ cells, $p<$ $0.05)$.

It is conceivable that $\mathrm{M} \beta \mathrm{CD}$-treatment enhances exocytotic release through an non-specific increase in cytosolic free $\mathrm{Ca}^{2+}$ concentration $\left(\left[\mathrm{Ca}^{2+}\right]_{\mathrm{i}}\right)$, a stimulus for regulated exocytosis $[14,38,65]$, thus elevating fusion pore conductance in both cell types $[65,66]$. Therefore, we measured changes in $\left[\mathrm{Ca}^{2+}\right]_{\mathrm{i}}$ in lactotrophs and astrocytes during the exposure to $10 \mathrm{mM} \mathrm{M} \beta \mathrm{CD}$ or $10 \mathrm{mM}$ cholesterol-replenishing solution. During $M \beta C D$ treatment $\left[\mathrm{Ca}^{2+}\right]_{\mathrm{i}}$ increased in lactotrophs but decreased in astrocytes (Figure S3). Therefore, cholesterol depletion may likely promote vesicle discharge by affecting the fusion pore properties directly as proposed in an in-vitro system [31], consistent with documented presynaptic effects of $\mathrm{M} \beta \mathrm{CD}$ on neurons, unrelated to cholesterol chelation [67].

\subsection{A theoretical description of cholesterol-mediated fusion pore properties}

We asked how fusion pore regulation can be mediated by the vesicle cholesterol transfer to the PM (Fig. 3). One possibility is that, at least in part, this is due to flip-flop transfer of cholesterol between the PM leaflets. This mechanism was found to be associated with membrane bending [68], which occurs during fusion pore formation. However, if the flip-flop mechanism dominates fusion pore regulation, then vesicle cholesterol accumulation in lysosomal storage disease [69] may not occur due to its depletion from vesicles. Therefore, based on the membrane elasticity theory [70] we developed a model (see Fig. 5), describing the mechanism of cholesterol transfer between the vesicle and the PM through a highly curved membranous fusion pore (Figs. 5A and $5 \mathrm{~B})$. At the fusion pore orifice, a shift in the membrane curvature occurs, from a negative curvature in the luminal face of the vesicle membrane to a positive one at the fusion pore orifice. The change in the curvature affects the properties of the PM [71], forming at quasi-equilibrium a boundary for membrane molecules with negative spontaneous curvature, such as cholesterol, to be transferred from the vesicle membrane to the PM. This generates tension [19] and creates a force, constricting the fusion pore diameter (Fig. 5C, black arrows). As the fusion pore diameter at steady state depends on the vesicle diameter [8-10], this model also considers this relationship (Fig. 5D). Details of this model are provided in the Supplementary Information (Figure S5).

In brief, line tension at the fusion pore creates a force constricting the latter (Fig. 5C; Supplementary Info., eq. 5). This force is proportional to the difference between cholesterol concentrations in the vesicle membrane and the PM (Supplementary Info., eq. 6). Owing to anisotropic structure and negative spontaneous curvature, cholesterol determines the bending elasticity of the membrane and preferentially accumulates in membrane domains with relatively high local negative curvature [8, 72]. Therefore, cholesterol density in the membrane is larger in more negatively curved membranes [71], i.e. at smaller fusion pore radii with respect to the secretory vesicle size (Supplementary Info, eq. 7). By simulating the influence of the difference in cholesterol concentrations between the vesicle membrane and the PM, we demonstrate a linear dependence of fusion pore radius on vesicle radius (Fig. 5D; 
A

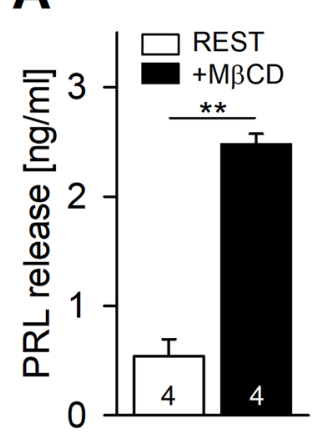

E
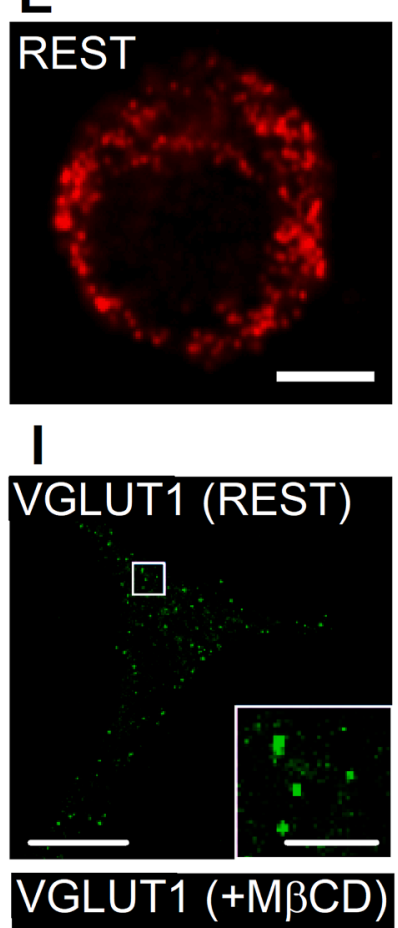

B

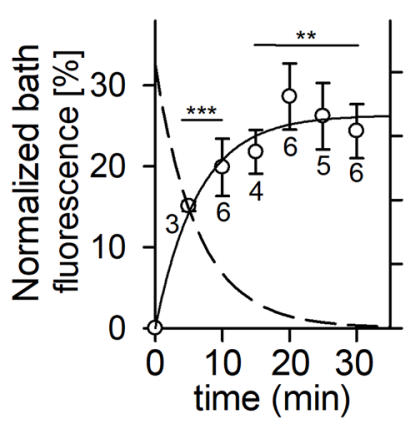

F

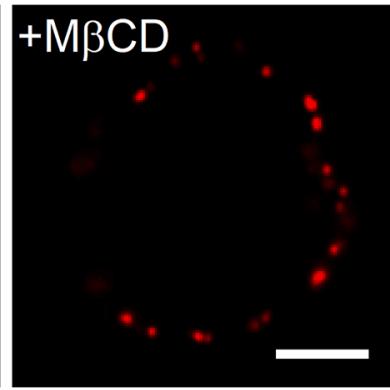

J
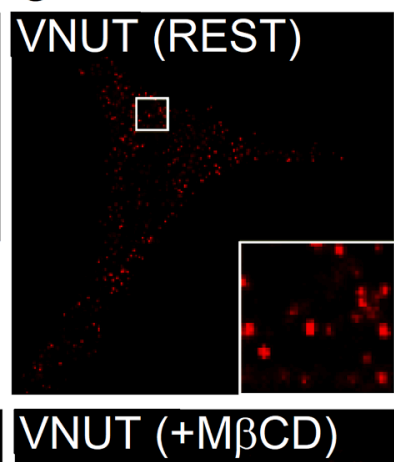
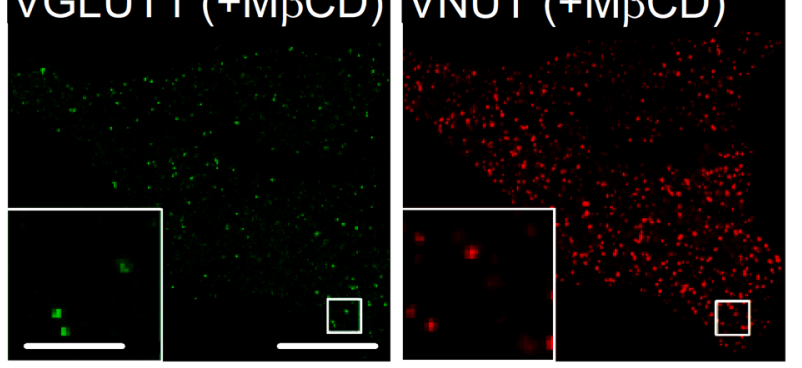

C

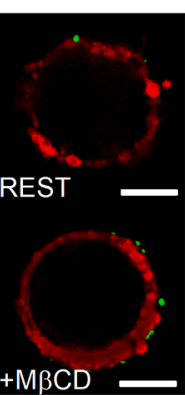

G
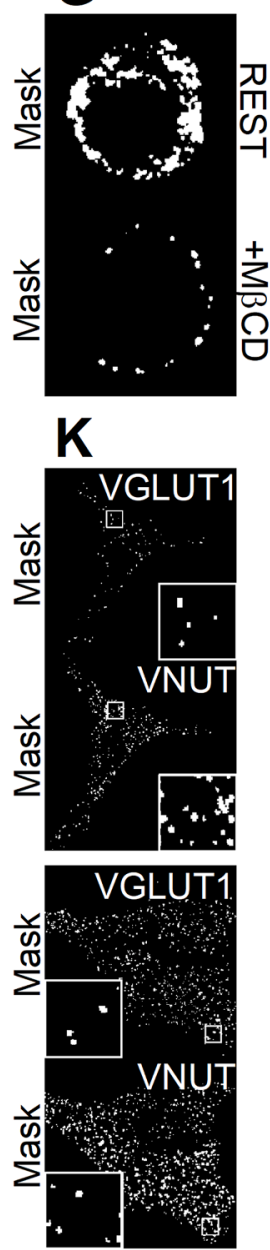

D

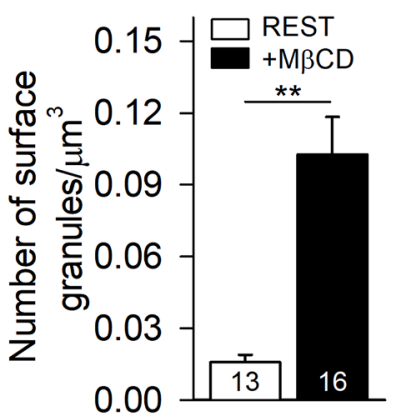

H

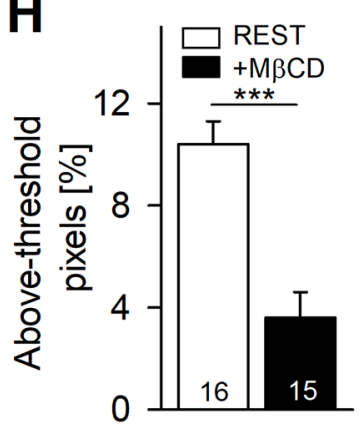

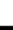
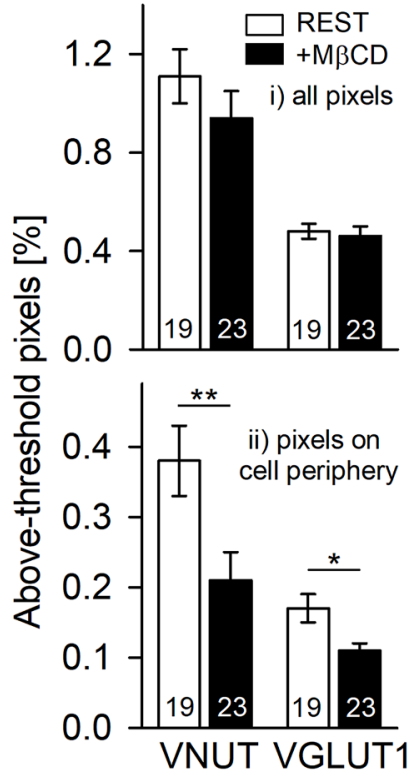

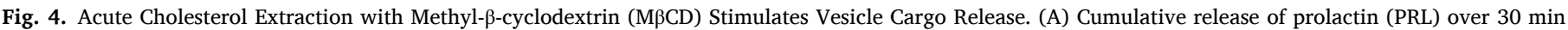

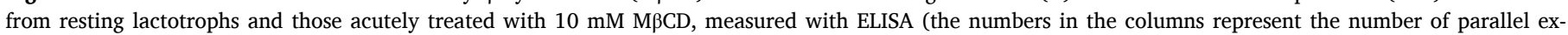

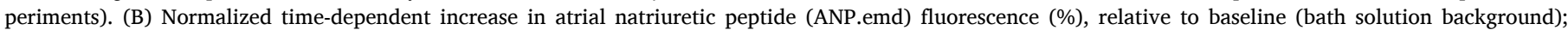

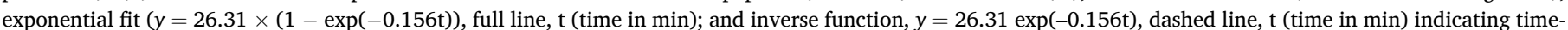

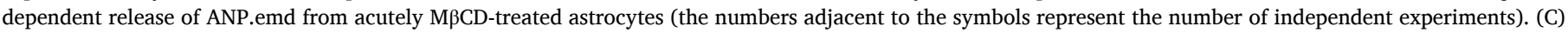

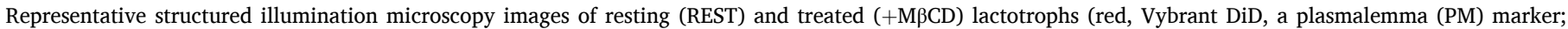

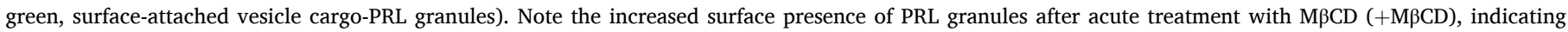

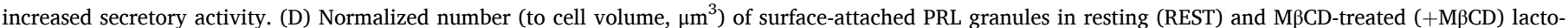

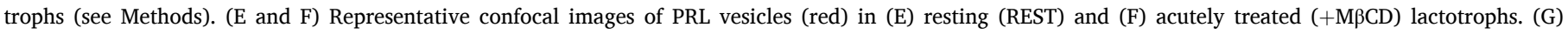

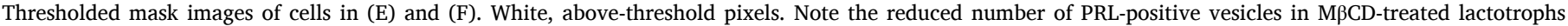

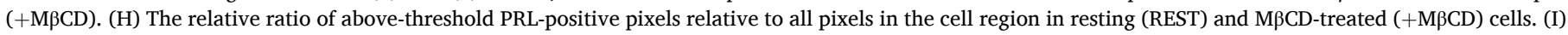

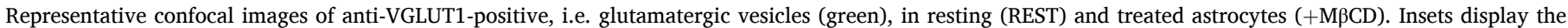

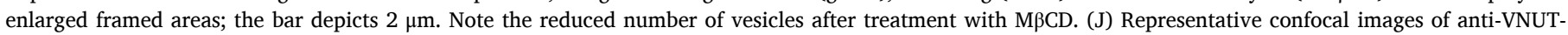

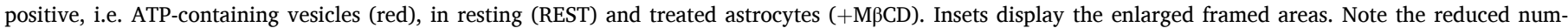

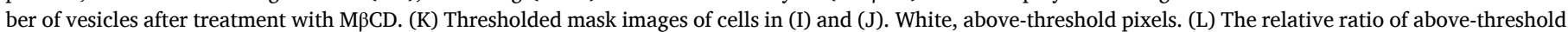

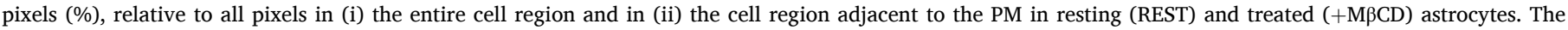

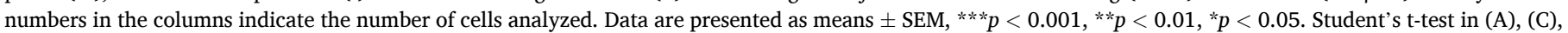
$(\mathrm{H})$, and (L); one-sample Student's $t$-test versus 0 in (D). Scale bars, $3 \mu \mathrm{m}$ in (B), (E) and (F); $10 \mu \mathrm{m}$ (inset, $2 \mu \mathrm{m})$ in (I) and (J). 
A

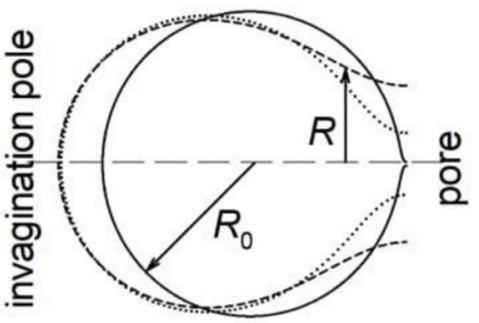

C

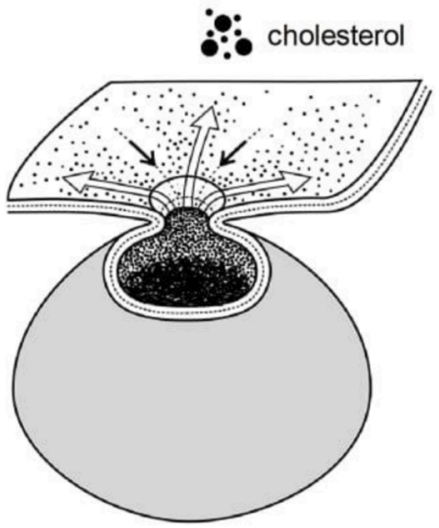

B

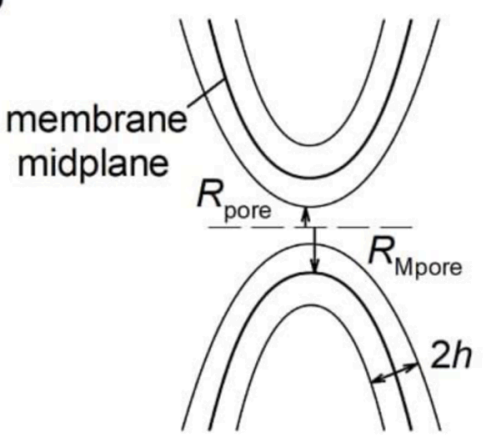

D

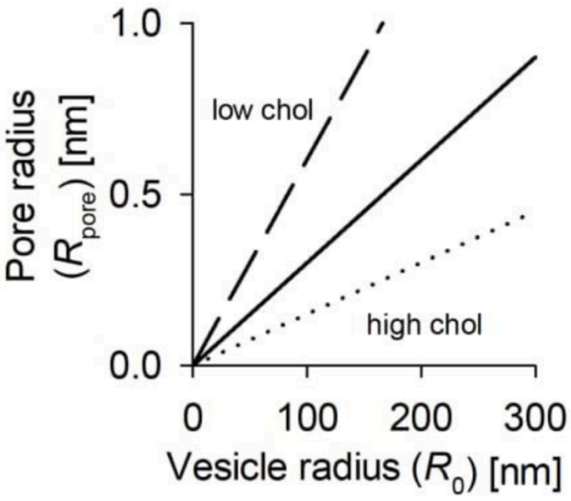

Fig. 5. Prediction of Fusion Pore Radius as a function of Vesicle Radius at Different Cholesterol Concentrations Details of Theoretical model are in Supplemental Information. (A) Longitudinal cross-sections of characteristic invagination shapes (secretory vesicle connected by the fusion pore to the $P M$ ) at a given invagination area $\left(A=4 \pi \mathrm{R}_{0}^{2}\right.$ ) for $\mathrm{R}_{0} \mathrm{c}_{\mathrm{p}}=1.003$ (full line), 1.045 (dotted line), and 1.099 (dashed line); $\mathrm{R}_{0}$ denotes the vesicle radius, $R$ denotes the distance of the vesicle membrane from the symmetry axis.

(B) Scheme of the fusion pore, where $R_{\text {pore }}$ denotes the fusion pore radius and $\mathrm{R}_{\mathrm{Mpore}}$ is the radius of the membrane midplane at the fusion pore.

(C) A scheme of the fusion pore and processes taking place when the fusion pore opens. Vesicle cholesterol density is depicted to be very high in the vesicle membrane as well as in the vesicle lumen, likely part of a crystalline array with hormones. Cholesterol in the intraluminal negatively curved face of the vesicle membrane exits the vesicle through the fusion pore. However, the positive membrane curvature at the fusion pore orifice at quasi-equilibrium conditions represents a boundary/barrier for cholesterol exiting the vesicle into the outer leaflet of the PM. The white arrows denote the direction of cholesterol transfer from the vesicle toward the outer leaflet of the PM. This creates tension and a radial force (tips of the black arrows), keeping the fusion pore radius constricted. Cholesterol molecules transferred from the vesicle membrane mix with cholesterol-rich rafts in the outerleaflet of the PM. See Supplementary material for the development of the model.

(D) Dependence of the fusion pore radius $\left(R_{\text {pore }}\right)$ as a function of the vesicle radius $\left(\mathrm{R}_{0}\right)$ for the ratio $\beta / \mathrm{E}_{0}=$ $6 \times 10^{-3}$ (dashed line), $3 \times 10^{-3}$ (full line), and $1.5 \times$ $10^{-3}$ (dotted line) (Supplementary information, eqs. 6 and 7 , where constants $\mathrm{E}_{0}, \beta$, and $\alpha$ are defined) at relatively large $\alpha$, representing low, normal, and high

cholesterol (chol.) in the PM, respectively.

Supplementary Info., eq. 9). The slope of this relationship is determined by cholesterol-related parameters (Supplementary Info.). Increased versus reduced cholesterol content is predicted to result in a relatively narrow (i.e. low $G_{p}$ ) versus wide fusion pore radius (i.e. high $G_{p}$ ), respectively (Fig. 5D).

\subsection{Increased fusion pore conductance in cells with lowered cholesterol}

To verify the role of cholesterol in determining the fusion pore properties according to the model (Fig. 5 and Supplementary Info.), unitary exocytotic events were monitored by high-resolution $\mathrm{C}_{\mathrm{m}}$ measurements in lactotrophs and astrocytes under control, cholesteroldepleted, and cholesterol-replenished conditions (Table S2).

In controls, $\sim 64 \%$ of patch-clamped lactotrophs ( 52 of 81 ) exhibited upward steps in $\mathrm{C}_{\mathrm{m}}$, and $95 \%(n=1354)$ of those were reversible, meaning that a discrete upward step in $\mathrm{C}_{\mathrm{m}}$ was followed by a downward step of similar amplitude within $3 \mathrm{~s}$ [66] (Fig. 6A). Similar reversible events were observed in astrocytes (Fig. 6B). These consecutive steps in $\mathrm{C}_{\mathrm{m}}$ reflect transient fusion pore openings [7, 14], whereas irreversible upward steps in $\mathrm{C}_{\mathrm{m}}$ (i.e. full fusion, Fig. $6 \mathrm{~A}$ ) represent a complete merger of the vesicle membrane with the PM [7]. Similar to a previous study [20], pretreatment with $10 \mathrm{mM} M \beta C D$ (Table S1) decreased the frequency of reversible fusion pore openings (Fig. 6C and 6D, Table S2). In cholesterol-replenished lactotrophs and astrocytes, the frequency of reversible fusion pore events was significantly increased (Fig. 6C and 6D). In lactotrophs the frequency of irreversible events was not affected by cholesterol depletion; however, after cholesterol replenishment, the frequency of irreversible events decreased slightly (Table S2). However, in astrocytes irreversible events decreased with cholesterol depletion and tended to increase upon cholesterol replenishment (Table S2).

The probability of observing a fusion pore in the open state, calculated as the sum of all dwell times of reversible $\mathrm{C}_{\mathrm{m}}$ events divided by the time of the recording, decreased significantly after cholesterol depletion $(p<0.01$; Fig. 6E) and was partially, yet significantly, restored after cholesterol replenishment to $0.86 \%$ ( $p<0.01$ versus control; Table S2). If a reversible $\mathrm{C}_{\mathrm{m}}$ event exhibits a projection from the imaginary (Im) to the real $(R e)$ part of the admittance signal (see Fig. 6A), then the $G_{p}$ can be calculated [41]. Such $C_{m}$ events comprised $15 \%$ of all events in control (203 of 1354 events), 11\% in cholesterol-depleted cells (44 of 400 events), and $28 \%$ in cholesterol-replenished cells (249 of 877 events; Fig. 6G), indicating that the probability of detecting events where $G_{p}$ can be measured depends on cholesterol. Assuming that the process evolves slowly enough for the system to remain in an internal equilibrium, the fusion pore length is stable [40]. Our experimental settings allowed the detection of fairly narrow diameter fusion pores (0.3-3 nm) [8]; therefore, the decrease in the relative occurrence of projected $\mathrm{C}_{\mathrm{m}}$ events in $\mathrm{M} \beta \mathrm{CD}$-treated cells indicated that cholesterol extraction promoted the formation of fusion pores with increased $G_{p}$ (i. e. wider diameters), which were no longer detectable as a projection between the $I m$ and $R e$ parts of the admittance signals. Consistent with this, the $G_{p} / C_{v}$ ratio, in which the fusion pore conductance $\left(G_{p}\right)$ is normalized to the vesicle size $\left(\mathrm{C}_{\mathrm{v}}\right)$, significantly increased in cholesterol-depleted cells (Fig. 6G, $p<0.05$; Table S2). In cholesterol-replenished lactotrophs, the $G_{p} / C_{v}$ ratio decreased significantly in comparison with controls $(p<0.01$; Table S2). The cholesterol-mediated changes in $\mathrm{G}_{\mathrm{p}} / \mathrm{C}_{\mathrm{v}}$ correlated with the measured 

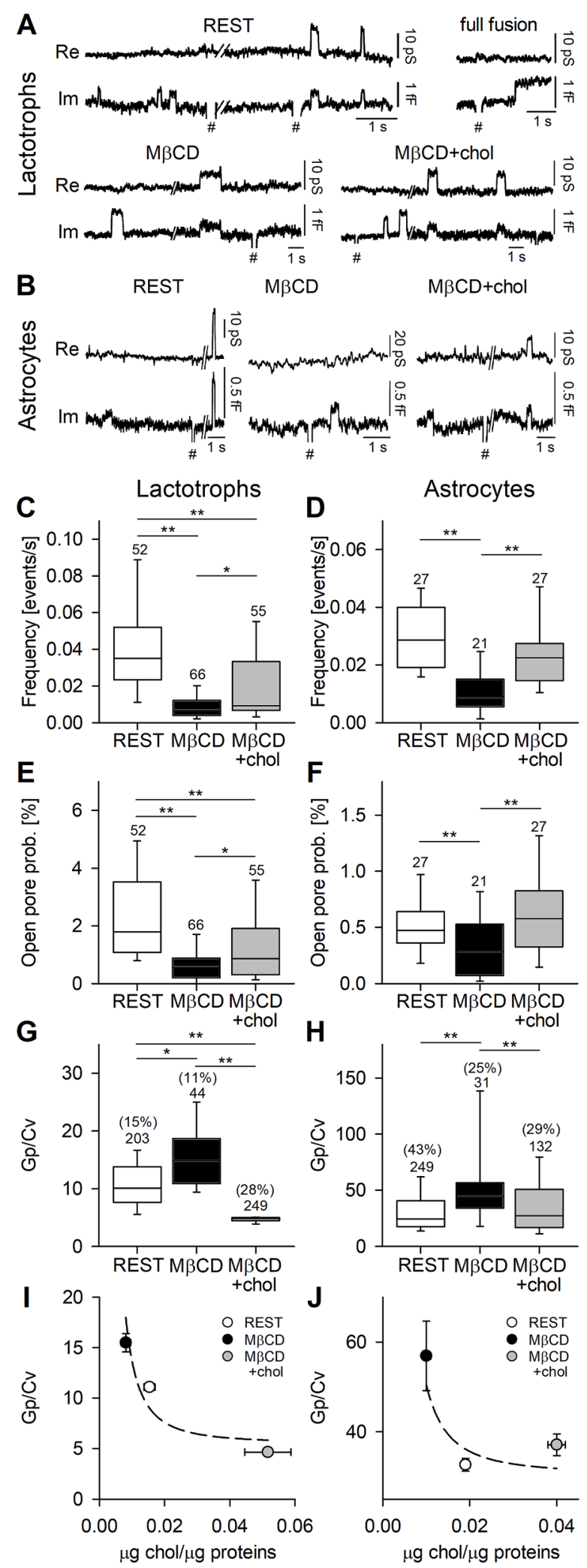

(caption on next page)
Fig. 6. Lowering Cholesterol Increases Fusion Pore Conductance, Rescued by Cholesterol Replenishment. (A) Representative patch-clamp cell-attached membrane recordings of $R e$ (real) and Im (imaginary) parts of admittance traces displaying reversible and irreversible $I m$ discrete steps (proportional to the membrane capacitance, $\mathrm{C}_{\mathrm{m}}$ ) with or without projections to $R e$, in resting (REST), cholesterol-extracted $(+\mathrm{M} \beta \mathrm{CD})$, and cholesterol-replenished $(\mathrm{M} \beta \mathrm{CD}+$ chol) lactotrophs.

(B) Representative $R e$ and $I m$ traces displaying reversible discrete Im steps with or without projections to $R e$ in resting (REST), $(+\mathrm{M} \beta C D)$, and $(\mathrm{M} \beta \mathrm{CD}+$ chol) treated astrocytes. Cells in the $+\mathrm{M} \beta \mathrm{CD}$ group in (A) and (B) were pretreated with $\mathrm{M} \beta \mathrm{CD}(10 \mathrm{mM}, 30 \mathrm{~min}$ ) before recordings. After cholesterol extraction and before recording, cholesterol was replenished (M $\beta C D+C h o l)$ with cholesterolreplenishing solution $(10 \mathrm{mM}, 30 \mathrm{~min})$.

(C and D) The frequency of reversible fusion events in resting (REST), $(+\mathrm{M} \beta \mathrm{CD})$, and $(\mathrm{M} \beta \mathrm{CD}+$ chol) lactotrophs (C) and astrocytes (D).

(E and F) Open (detectable) fusion pore probability in resting (REST), $(+\mathrm{M} \beta \mathrm{CD})$, and $(\mathrm{M} \beta \mathrm{CD}+\mathrm{chol})$ lactotrophs $(\mathrm{E})$ and astrocytes $(\mathrm{F})$.

( $G$ and $H$ ) Fusion pore conductance $\left(G_{p}\right)$ normalized to vesicle membrane capacitance $\left(\mathrm{C}_{\mathrm{v}}\right)\left(\mathrm{G}_{\mathrm{p}} / \mathrm{C}_{\mathrm{v}}\right)$ in resting (REST), $(+\mathrm{M} \beta C D)$, and $(\mathrm{M} \beta \mathrm{CD}+$ chol) treated lactotrophs $(\mathrm{G})$ and astrocytes $(\mathrm{H})$. The numbers in parentheses denote the percentage of Re projections to the Im trace, relative to all transient Im events.

(I-J) Normalized fusion pore conductance $\left(G_{p} / C_{v}\right)$, proportional to $\left(R_{\text {pore }} / R_{0}\right)^{2}$ (Supplementary material, eq. 7) versus normalized cholesterol concentration ( $\mu \mathrm{g} \mathrm{chol} / \mu \mathrm{g}$ protein), proportional to $\mathrm{E}_{1}$ (Supplementary material) in resting (REST), $(+\mathrm{M} \beta \mathrm{CD})$, and $(\mathrm{M} \beta \mathrm{CD}+\mathrm{chol})(\mathrm{I})$ lactotrophs and $(\mathrm{J})$ astrocytes. Fitted (to all data pairs) inverse square curve is of the form $\left(\mathrm{G}_{\mathrm{p}} / \mathrm{C}_{\mathrm{v}}\right)=\left(8.23 \times 10^{-4} \pm\right.$ $\left.\left.4.35 \times 10^{-5}\right) / \mathrm{x}^{2}\right)+(5.33 \pm 0.23), r=0.649$ (I) and

$\left.\left(\mathrm{G}_{\mathrm{p}} / \mathrm{C}_{\mathrm{v}}\right)=\left(2.00 \times 10^{-3} \pm 5.56 \times 10^{-4}\right) / \mathrm{x}^{2}\right)+(30.59 \pm 1.95), r=0.174(\mathrm{~J})$. The numbers above the box plots indicate the number of cells analyzed in (C)(F) and the number of transient Im events with Re projections in (G) and (H). Data are presented as medians with interquartile range $(\mathrm{C}-\mathrm{H})$ and means \pm SEM in (I and J). ${ }^{* *} p<0.01,{ }^{*} p<0.05$. ANOVA on ranks with Dunn's post-hoc test. \# denotes $10 \mathrm{fF}$ calibration.

cholesterol concentration (Table S1 and Fig. 6I).

Similar results were obtained in astrocytes. The frequency of reversible $C_{m}$ events significantly $(p<0.01)$ decreased in M $\beta C D$-treated cells and was restored $(p<0.01)$ in cholesterol-replenished cells (Fig. 6D). We observed a significant decrease in the frequency of irreversible events in cholesterol-depleted astrocytes, which was restored by cholesterol replenishment (Table S2). Moreover. the percentage of reversible events, in comparison with all events, decreased in cholesterol-depleted astrocytes and was restored by cholesterol replenishment (Table S2).

The probability of observing the fusion pore open state (Fig. $6 \mathrm{~F}$ ) was significantly reduced by cholesterol depletion ( $p<0.01$; Table S2). Cholesterol replenishment fully restored the open fusion pore probability in astrocytes, and partially in lactotrophs $(p<0.01$ vs. cholesteroldepleted cells; Table S2). Similar to the recordings in lactotrophs, there was a decrease in the reversible events with projections (representing relatively narrow fusion pore diameters) from the $\mathrm{Im}$ to $\mathrm{Re}$ parts of the admittance [41], from $43 \%$ ( 249 of 576 events) in controls to $25 \%$ (31 of 122 events) in cholesterol-depleted astrocytes; this increased to $29 \%$ (132 of 462 events) with cholesterol replenishment. Accordingly, the $\mathrm{G}_{\mathrm{p}} / \mathrm{C}_{\mathrm{v}}$ ratio (Fig. $6 \mathrm{H}$ ) in astrocytes increased significantly after cholesterol depletion $(p<0.01)$ and was fully restored by cholesterol replenishment ( $p<0.01$ versus cholesterol-depleted cells; Table S2). The cholesterol-dependent $\mathrm{G}_{\mathrm{p}} / \mathrm{C}_{\mathrm{v}}$ correlated with the measured cholesterol (Fig. 6J).

These results demonstrate that relatively high cholesterol levels reduce $G_{p}$, whereas cholesterol depletion elevates it, consistent with our theoretical predictions (Fig. 5D) as further confirmed in Figure S4, 
which display the relationship between the fusion pore diameter (estimated from $\mathrm{G}_{\mathrm{p}}$ ) and the vesicle diameter (determined from $\mathrm{C}_{\mathrm{v}}$; lines fitted to eq. 9: Supplementary Info.).

\subsection{Reduced fusion pore conductance in vesicles of $\mathrm{Npc1}^{-/-}$fibroblasts}

Cholesterol-dependent regulation of the fusion pore can be modified in pathology. To address this question we used mouse fibroblast cellular

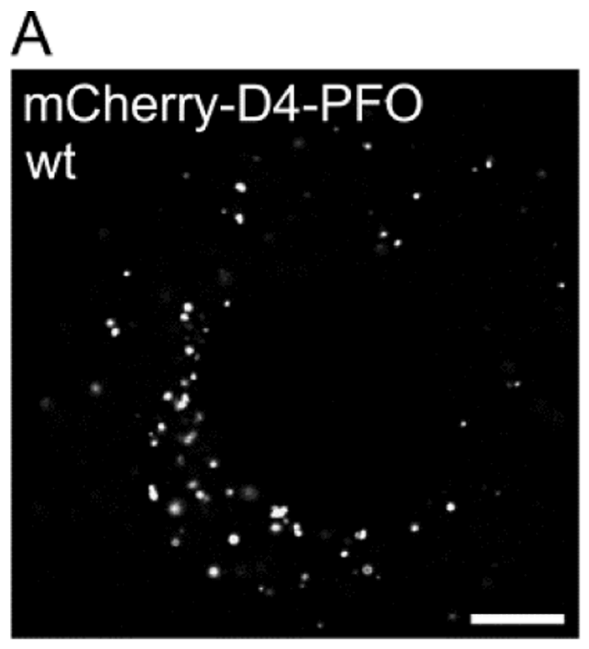

B

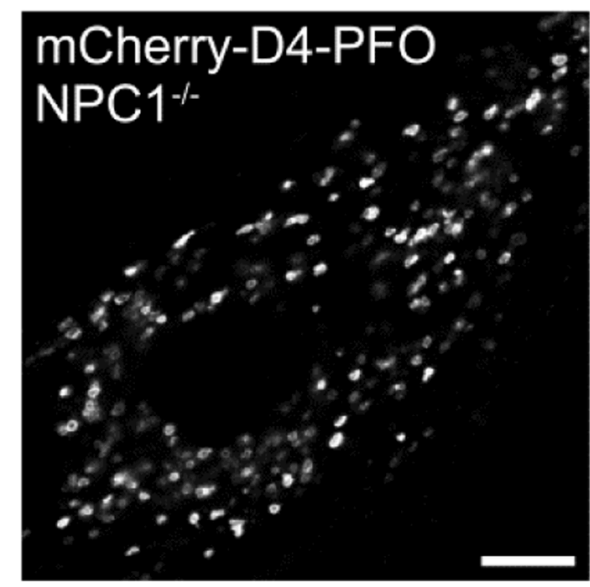

C

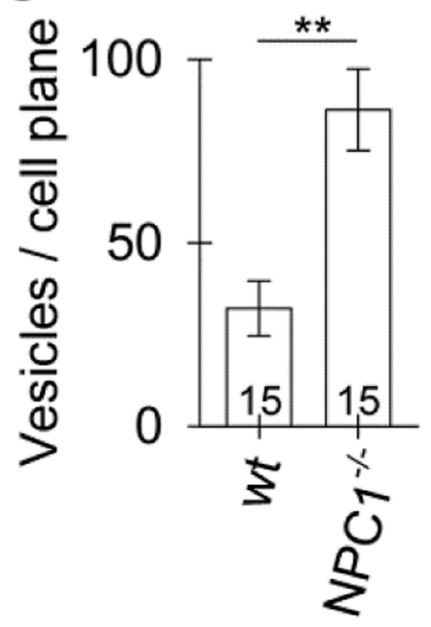

D

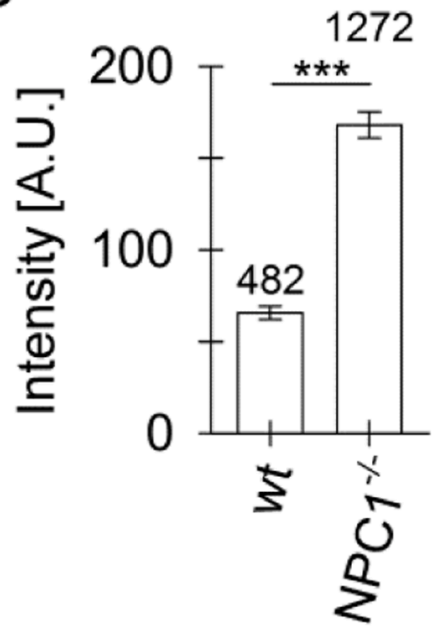

$E$ wt

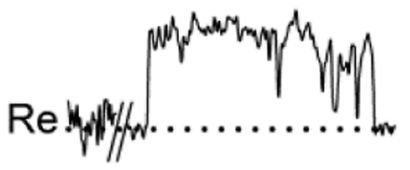

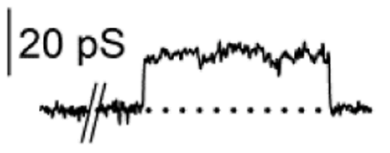

$1 \mathrm{~s}$

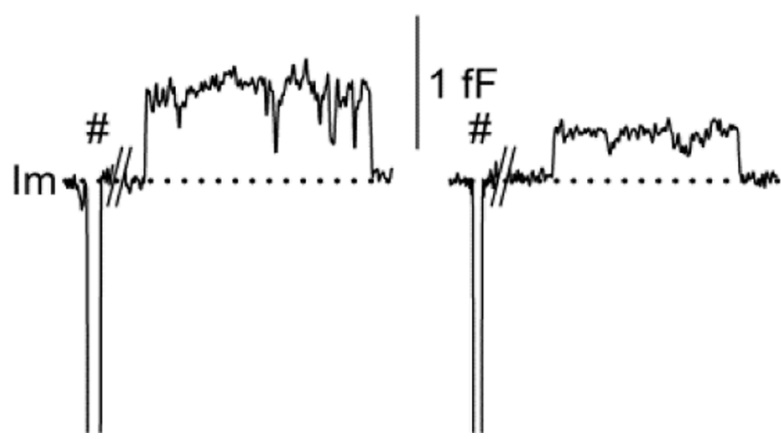

Fig. 7. Increased Cholesterol Content in Fibroblasts Lacking Npc1 is Associated with Reduced Fusion Pore Conductance. (A and B) Confocal images of fixed wildtype (wt) and $\mathrm{Npc1}^{-/-}$fibroblasts labeled with the cholesterol-specific membrane-binding domain of perfringolysin $\mathrm{O}$ (mCherry-D4) reveal numerous vesiclelike structures in the $\mathrm{Npc1}^{-/-}$versus wt cells (scale bar, $10 \mu \mathrm{m})$

(C and D) Number (mean \pm SEM) of mCherry-D4positive vesicles per cell plane (C) and the cumulative vesicle fluorescence intensity (D) in wt and $\mathrm{Npc1}^{-/-}$ fibroblasts. The numbers at the bottom and top of the bars indicate the number of cell images and vesicles analyzed, respectively; ${ }^{* *} p<0.01,{ }^{* * *} p<0.001$ versus respective comparisons (Mann-Whitney U test).

(E) Electrophysiological recordings of real ( $R e$, top) and imaginary (Im, bottom) parts of the admittance signals in vesicles transiently interacting with the PM in wt and $\mathrm{Npc1}^{-/-}$fibroblasts. Dotted lines indicate baseline level; \# denote calibration pulses.

(F) Fusion pore conductance $\left(G_{p}\right)$ normalized to vesicle capacitance $\left(\mathrm{C}_{\mathrm{v}}, \mathrm{G}_{\mathrm{p}} / \mathrm{C}_{\mathrm{v}}\right)$ is reduced in $N p c 1^{-/-}$fibroblasts. The numbers at the bottom of the bars indicate the number of vesicles analyzed. ${ }^{* *} p<0.01$ (MannWhitney U test). 
model of lysosomal storage disease, in which the Niemann-Pick Disease Type C1 (Npc1) protein, the vesicle cholesterol transporter, is deleted $[69,73]$. Confocal microscopy of fixed cultured cells revealed abundant D4-positive vesicles in the cytoplasm of $\mathrm{Npc1}^{-/-}$cells (Figs. 7A and 7B). The number of D4-positive vesicles (per cell plane) was $\sim 2.7$ fold higher in $N p c 1^{-/-}$cells $(p<0.01, n=15)$ compared with wild-type fibroblasts ( $n=15$; Fig. $7 \mathrm{C}$ ), and the cumulative fluorescence intensity of D4-positive vesicles was $\sim 2.6$-fold higher in $N p c 1^{-/-}(p<0.001)$ than in wild-type fibroblasts (Fig. 7D). These data indicate that fibroblasts lacking functional Npc1 accumulate cholesterol in vesicles, presumably in late endosome/lysosomes [73], consistent with a previous study [74].

High-resolution $C_{m}$ recordings revealed that $G_{p}$, normalized to vesicle capacitance $\left(\mathrm{G}_{\mathrm{p}} / \mathrm{C}_{\mathrm{v}}\right)$, decreased from $98 \pm 5 \mathrm{pS} / \mathrm{fF}$ in controls $(n$ $=172)$ to $83 \pm 5 \mathrm{pS} / \mathrm{fF}$ in $N p c 1^{-/-}$fibroblasts $(n=116, p<0.01$, Fig. 7F), indicating that increased vesicle cholesterol in $\mathrm{Npc1}^{-/-}$fibroblasts reduces the $G_{p}$ of spontaneously fusing vesicles.

\section{Discussion}

In this study we asked whether the vesicle accumulation of cholesterol, often a consequence of inborn enzymatic deficiency common to a large group of lysosomal storage diseases [3], affects the function of the fusion pore, an intermediate of multiple-staged exocytosis [6]. Fusion begins with the apposition of membranes destined to fuse, and terminates with (1) destabilization of the membranes in contact, (2) coalescence of the two membrane surfaces and (3) re-stabilization of the newly fused membranes. These stages are considered to occur within microseconds to maintain the cell's integrity. This view developed from purely lipidic models, while fundamentally appropriate overall, introduced the idea that once the fusion pore is formed it must widen abruptly (full-fusion exocytosis) [6]. However, recent experiments in native cell systems revealed that the fusion pore, once established, enters a stable, dynamically regulated state, with diameters ranging between sub-nanometers and several hundred nanometers $[10,65]$. These pores can undergo either reversible constriction or closure to limit vesicle discharge or full widening (full-fusion exocytosis) to boost vesicle discharge [75]. It is generally acknowledged that these modulatory transitions are regulated by proteins, including actin, required for widening of fusion pores with diameters exceeding $100 \mathrm{~nm} \mathrm{[10],} \mathrm{where}$ the vesicle membrane may transfer to the PM, manifested as vesicle shrinkage [76-78]. This shrinkage is influenced by the osmotic pressure difference between the intracellular and the extracellular solution and actin-mediated membrane tension to reel in the membrane of the fusing vesicles [77, 78]. Recently, G-protein coupled receptors were also found to control the fusion pore [79]. Here we asked whether cholesterol affects the native fusion pore, in particular the sub-nanometer one [65]. There has been interest in how cholesterol initiates and regulates the stages of regulated exocytosis [16, 20, 22-24, 26, 27, 35, 80]; however, direct evidence defining the dynamics of single fusion pore stages in living secretory cells is missing. Here the role of a cholesterol-dependent mechanism that reduces the fusion pore conductance is established (Figs. 6 and 7). This new mechanism of fusion pore regulation posits that vesicle cholesterol transfer to the PM is relevant for lysosomal storage diseases.

Four lines of evidence support this conclusion. First, the spontaneous negative curvature of cholesterol, a structural stress factor [6], likely promotes the first step of the membrane merger [16, 21, 28], and is also essential during stages after the fusion pore is formed [75]. Upon stimulating regulated exocytosis, the fusion pore is formed, through which vesicle cholesterol exits and subsequently mixes with the PM. In some cells, cholesterol accumulates in lysosomes, especially in diseased states $[69,73]$, and in the vesicle membranes of secretory cells [55, 80]. Consistent with these previous studies, we found that PRL-storing vesicles contain cholesterol (Fig. 1). PRL is discharged from vesicles through regulated exocytosis [58], while vesicle cholesterol is transferred to the PM. Stimulation of regulated exocytosis reduced the density and size of the mCherry-D4-PFO (D4) labeled cholesterol-rich PM domains (Fig. 2), reflecting the PM-targeted delivery of vesicle cholesterol paralleling the hormone discharge. The surface PM deposits of fluorescently labeled hormone from vesicles correlated with an increase in the PM cholesterol D4-domains (Fig. 3). To exit vesicles, hormones and cholesterol require a gradient; hormones are released into the extracellular space and cholesterol into the PM. Cholesterol exiting the vesicle to the PM may also regulate the fusion pore, as tested in subsequent experiments.

The second line of evidence is that cholesterol depletion stimulates hormone release independent to changes in $\left[\mathrm{Ca}^{2+}\right]_{\mathrm{i}}$ (Figure S3). Exposing cells to $\mathrm{M} \beta \mathrm{CD}$ while monitoring amperometric discharge of vesicle cargo [23, 24] revealed that cholesterol enrichment increased the persistence of the pre-foot spike of the amperometric signal, which indirectly represents the leakage of vesicle cargo through a narrow fusion pore before the pore dilates for full exocytotic release [81]. We monitored vesicle discharge in electrically excitable pituitary lactotrophs and in electrically non-excitable astrocytes, which exhibit distinct $\mathrm{Ca}^{2+}$ sources [14, 38, 82]. Vesicle cargo, labeled by immunofluorescence in lactotrophs or by the expression of fluorescently tagged peptide in astrocytes, was secreted following M $\beta C D$ treatment (Fig. 4). However, at steady state, in the absence of stimulation, direct monitoring of fusion pore conductance, a measure of fusion pore diameter, revealed that cholesterol depletion increases while cholesterol enrichment reduces the fusion pore conductance in both cell types (Fig. 6). The opposite effects of $\mathrm{M} \beta \mathrm{CD}$ application on $\left[\mathrm{Ca}^{2+}\right]_{\mathrm{i}}$ in astrocytes and lactotrophs (Fig. 4) reflects distinct $\mathrm{Ca}^{2+}$ sources regulating exocytosis in these cells. Lactotrophs are electrically excitable endocrine pituitary cells, firing action potentials and expressing voltage-gated $\mathrm{Ca}^{2+}$ channels [83], which regulate exocytosis [14]. In addition, several regulators of prolactin secretion, including TRH, control the release of $\mathrm{Ca}^{2+}$ from intracellular stores [84]. In contrast, secretion from astrocytes arises mainly from delivery of $\mathrm{Ca}^{2+}$ from intracellular stores to the exocytotic apparatus [85-88].

Cholesterol replenishment is only mildly effective in lactotrophs compared to its effect on astrocytes (Fig. 6; Table S2). This arguably reflects the function of the studied cell types. Lactotrophs are specialized endocrine cells for the release of hormone prolactin. Astrocytes also release a number of chemical messengers by regulated exocytosis [38, 89, 90], however their main physiological function is to provide homeostatic support for nervous tissue; for example by providing energy substrate in the form of L-lactate [91] to support learning and memory formation [92]. Moreover, astrocytes are the main provider of cholesterol needed for neuronal and synaptic plasticity in the CNS [93].

Third, the molecular conformation and physicochemical properties of cholesterol affect membrane curvature, including the initial membrane merger step of regulated exocytosis $[6,8,28,94]$. The walls of the open fusion pore represent a highly curved structure contacting the PM. This orifice represents, at quasi-equilibrium, a boundary across which the cholesterol gradient between the vesicle and the PM exerts tension. Due to this tension, a force is generated, which prevents fusion pore widening (Fig. 5C). The force at the orifice of the fusion pore depends on the difference in cholesterol concentration between the vesicle and the $\mathrm{PM}$, driving cholesterol redistribution from vesicle into the PM (Figs. 2 and 3). Based on this, a theoretical model of how cholesterol controls the fusion pore diameter was developed (Suppl. Info.) by using the spontaneous-curvature model $[70,95]$, the key concept in understanding membrane mechanics. To stabilize the fusion pore diameter the energy of membrane adhesion was considered to play a prominent role [96]. In our model, bending energy was considered to be essentially influenced by vesicle size [8-10]. Vesicles represent a source of membrane material, such as cholesterol, contributing to the fusion pore stabilization through a force constricting the fusion pore. This force depends linearly on the local cholesterol availability (Suppl. Info., eq. 6; see also the direction of the forces depicted in Fig. 5C). We estimated the force at the fusion pore $\left(\gamma_{\text {pore }}\right)$ from the dimensionless transfer shear force $\left(\Gamma_{\text {pore }}\right)$ in the radial direction: $\Gamma_{\text {pore }}=R_{0} \gamma_{\text {pore }} / 8 \pi k_{c}$, where $R_{0}$ is the 
vesicle radius and $k_{c}$ is the bending modulus (Suppl. Info.). For a typical value of $\Gamma_{\text {pore }}=0.4$ (Figure S5B), using $R_{0}=100 \mathrm{~nm}$ and $k_{c}=20 \mathrm{k}_{\mathrm{B}} \mathrm{T}$ [97], the estimate for $\gamma_{\text {pore }}$ is $80 \mathrm{pN}$, essentially the same as the value reported for the fusion pore in chromaffin cells [78]. A similar range of forces affecting the fusion pore can be predicted by considering the interactions between SNARE proteins at post-fusion stages of exocytosis [98].

The validity of this model is corroborated by experimental data (Figs. 6I and 6J), showing that normalized fusion pore conductance $\left(\mathrm{G}_{\mathrm{p}} /\right.$ $\mathrm{C}_{\mathrm{v}}$ ) correlates with cholesterol content, indicating that changes in cellular cholesterol content can regulate the vesicle fusion pore.

Fourth, we demonstrate that disease-related increase in vesicle cholesterol affects the fusion pore (Fig. 7F) consistent with predictions by the model (Fig. 5D). In Niemann-Pick disease type C1 mutations in NPC1, a protein involved in lysosome-ER contact site formation and therefore indirectly facilitating sterol transport [99, 100], leads to an accumulation of vesicle cholesterol, characteristic to lysosomal storage diseases [69, 73]. In $\mathrm{Npc1}^{-/-}$fibroblasts vesicles contained on average $\sim 2$-times more cholesterol, than in healthy cells (Fig. 7A-D). High-resolution measurements of fusion pore conductance, normalized to vesicle capacitance $\left(\mathrm{G}_{\mathrm{p}} / \mathrm{C}_{\mathrm{v}}\right)$, revealed fusion pore conductance decrease by over $15 \%$ in these fibroblasts (Fig. 7). These data demonstrate that the disease-related increase in vesicle cholesterol in $\mathrm{Npc1}^{-/-}$ fibroblasts affects the fusion pore conductance, which arguably hinders the vesicle cargo release. The contribution this makes to the pathophysiology of NPC therefore merits further investigation.

\section{Conclusions}

In conclusion, this paper demonstrates that stimulation of exocytosis leads to the transfer and subsequent mixing of vesicle cholesterol with the PM. Cholesterol transfer instigates a reduction in fusion pore conductance through a force generated at the orifice of the fusion pore where the change in the membrane curvature takes place. At quasiequilibrium conditions this forms a boundary for membrane molecules with negative spontaneous curvature, such as cholesterol, to be transferred from the vesicle membrane to the plasma membrane, generating tension and creating a force constricting the fusion pore diameter. This represents a new foundation for understanding the nature of this ubiquitous membrane intermediate of eukaryotic cells in health and disease, including LSD.

\section{Author agreement statement}

Authors declare that this manuscript is original, has not been published before and is currently not being considered for publication elsewhere. Authors confirm that the manuscript has been read and approved by all authors and that there are no other persons who satisfied the criteria for authorship but are not listed. Authors further confirm that the order of authors listed in the manuscript has been approved by all of us.

\section{CRediT authorship STATEMENT}

B.R., A.G., M.L., U.G., S.T.B., E.L., M.B. and M.S. carried out electrophysiology and imaging experiments and performed data analysis. P. S.A. and J.R.C. carried out the lipidomic analysis after cholesterol depletion in cells. A.Šs. and G.A. developed and prepared the D4 cholesterol fluorescent marker. F.M.P. provided the Npc1 deficient and control fibroblasts. B.B. developed the cholesterol-mediated radial force membrane fusion pore constriction model. R.Z., J.R.C., B.B., J.J., A.V., M.K., and M.S. discussed the design of the experiments. R.Z. directed and wrote the manuscript with help from all authors. Further information and requests for resources and reagents should be directed to and will be fulfilled by the Lead Contact, Robert Zorec (robert.zorec@mf. uni-lj.si).

\section{References cited in Supplementary materials}

$[101,102,103,104,105,106]$.

\section{Declaration of competing interests}

The authors declare that they have no known competing financial interests or personal relationships that could have appeared to influence the work reported in this paper.

\section{Availability of data and material}

All data generated or analysed during this study are included in this published article and its Supplementary Information files or are available from the corresponding author on

\section{Acknowledgments}

This work was supported by grants from the Slovenian Research Agency (P3 310, P1-0055, J3 4051, J3 4146, L3 3654; J3 3236, J3 6790, J3 6789, J3 7605), CIPKEBIP, COST Nanonet, COST Mouse Ageing, COST CM1207 - GLISTEN and CELSA. JRC acknowledges the support of the NHMRC (Australia; APP1065328) and Brock University. F.M.P. is a Royal Society Wolfson Research Merit Award holder and a Wellcome Trust Investigator in Science. Claire Smith from the Platt Lab is acknowledged for the technical expertise with the Npc1 knockout and control fibroblasts.

\section{Supplementary materials}

Supplementary material associated with this article can be found, in the online version, at doi:10.1016/j.ceca.2021.102503.

\section{References}

[1] N. Brose, A. Brunger, D. Cafiso, E.R. Chapman, J. Diao, F.M. Hughson, M. B. Jackson, R. Jahn, M. Lindau, C. Ma, J. Rizo, Y.K. Shin, T.H. Sollner, L. Tamm, T.Y. Yoon, Y. Zhang, Synaptic vesicle fusion: today and beyond, Nat. Struct. Mol. Biol. 26 (2019) 663-668.

[2] A. Fraldi, F. Annunziata, A. Lombardi, H.J. Kaiser, D.L. Medina, C. Spampanato, A.O. Fedele, R. Polishchuk, N.C. Sorrentino, K. Simons, A. Ballabio, Lysosomal fusion and SNARE function are impaired by cholesterol accumulation in lysosomal storage disorders, EMBO J. 29 (2010) 3607-3620.

[3] G. Parenti, G. Andria, A Ballabio, Lysosomal storage diseases: from pathophysiology to therapy, Annu. Rev. Med. 66 (2015) 471-486.

[4] W.D. Zhao, E. Hamid, W. Shin, P.J. Wen, E.S. Krystofiak, S.A. Villarreal, H. C. Chiang, B. Kachar, L.G. Wu, Hemi-fused structure mediates and controls fusion and fission in live cells, Nature 534 (2016) 548-552.

[5] S. Sharma, M. Lindau, The fusion pore, 60 years after the first cartoon, FEBS Lett. (2018).

[6] R.P. Rand, V.A. Parsegian, Mimicry and mechanism in phospholipid models of membrane fusion, Annu. Rev. Physiol. 48 (1986) 201-212.

[7] E. Neher, A Marty, Discrete changes of cell membrane capacitance observed under conditions of enhanced secretion in bovine adrenal chromaffin cells, Proc. Natl. Acad. Sci. U. S. A. 79 (1982) 6712-6716.

[8] J. Jorgacevski, M. Fosnaric, N. Vardjan, M. Stenovec, M. Potokar, M. Kreft, V. Kralj-Iglic, A. Iglic, R. Zorec, Fusion pore stability of peptidergic vesicles, Mol. Membr. Biol, 27 (2010) 65-80.

[9] A. Flasker, J. Jorgacevski, A.I. Calejo, M. Kreft, R. Zorec, Vesicle size determines unitary exocytic properties and their sensitivity to sphingosine, Mol. Cell. Endocrinol. 376 (2013) 136-147.

[10] W. Shin, L. Ge, G. Arpino, S.A. Villarreal, E. Hamid, H. Liu, W.D. Zhao, P.J. Wen, H.C. Chiang, L.G. Wu, Visualization of membrane pore in live cells reveals a dynamic-pore theory governing fusion and endocytosis, Cell 173 (2018) 934-945, e12.

[11] L. He, X.S. Wu, R. Mohan, L.G. Wu, Two modes of fusion pore opening revealed by cell-attached recordings at a synapse, Nature 444 (2006) 102-105.

[12] C.P. Grabner, T. Moser, Individual synaptic vesicles mediate stimulated exocytosis from cochlear inner hair cells, Proc. Natl. Acad. Sci. U. S. A. 115 (2018) 12811-12816.

[13] V.A. Klyachko, M.B. Jackson, Capacitance steps and fusion pores of small and large-dense-core vesicles in nerve terminals, Nature 418 (2002) 89-92. 
[14] R. Zorec, S.K. Sikdar, W.T. Mason, Increased cytosolic calcium stimulates exocytosis in bovine lactotrophs. Direct evidence from changes in membrane capacitance, J. Gen. Physiol. 97 (1991) 473-497.

[15] T. Lang, D. Bruns, D. Wenzel, D. Riedel, P. Holroyd, C. Thiele, R. Jahn, SNAREs are concentrated in cholesterol-dependent clusters that define docking and fusion sites for exocytosis, EMBO J. 20 (2001) 2202-2213.

[16] T. Rogasevskaia, J.R. Coorssen, Sphingomyelin-enriched microdomains define the efficiency of native $\mathrm{Ca}(2+)$-triggered membrane fusion, J. Cell Sci. 119 (2006) 2688-2694.

[17] P.L. Yeagle, Lanosterol and cholesterol have different effects on phospholipid acyl chain ordering, Biochim. Biophys. Acta 815 (1985) 33-36.

[18] D.J. Recktenwald, H.M. McConnell, Phase equilibria in binary mixtures of phosphatidylcholine and cholesterol, Biochemistry 20 (1981) 4505-4510.

[19] F. Julicher, R. Lipowsky, Shape transformations of vesicles with intramembrane domains, Phys. Rev. E Stat. Phys. Plasmas Fluids Relat. Interdiscip. Topics 53 (1996) 2670-2683.

[20] B. Rituper, A. Flasker, A. Gucek, H.H. Chowdhury, R. Zorec, Cholesterol and regulated exocytosis: a requirement for unitary exocytotic events, Cell Calcium 52 (2012) 250-258.

[21] M.A. Churchward, J.R. Coorssen, Cholesterol, regulated exocytosis and the physiological fusion machine, Biochem. J. 423 (2009) 1-14.

[22] M.A. Churchward, T. Rogasevskaia, J. Hofgen, J. Bau, J.R. Coorssen, Cholesterol facilitates the native mechanism of Ca2+-triggered membrane fusion, J. Cell Sci. 118 (2005) 4833-4848.

[23] N. Wang, C. Kwan, X. Gong, E.P. de Chaves, A. Tse, F.W. Tse, Influence of cholesterol on catecholamine release from the fusion pore of large dense core chromaffin granules, J. Neurosci. 30 (2010) 3904-3911.

[24] E.A. Cookson, I.L. Conte, J. Dempster, M.J. Hannah, T. Carter, Characterisation of Weibel-Palade body fusion by amperometry in endothelial cells reveals fusion pore dynamics and the effect of cholesterol on exocytosis, J. Cell Sci. 126 (2013) 5490-5499.

[25] B. Rituper, H.H. Chowdhury, J. Jorgacevski, J.R. Coorssen, M. Kreft, R. Zorec, Cholesterol-mediated membrane surface area dynamics in neuroendocrine cells, Biochim. Biophys. Acta 1831 (2013) 1228-1238.

[26] B.S. Stratton, J.M. Warner, Z. Wu, J. Nikolaus, G. Wei, E. Wagnon, D. Baddeley, E. Karatekin, B. O'Shaughnessy, Cholesterol increases the openness of SNAREmediated flickering fusion pores, Biophys. J. 110 (2016) 1538-1550.

[27] N. Najafinobar, L.J. Mellander, M.E. Kurczy, J. Dunevall, T.B. Angerer, J. S. Fletcher, A.S. Cans, Cholesterol alters the dynamics of release in protein independent cell models for exocytosis, Sci. Rep. 6 (2016) 33702.

[28] J.R. Coorssen, R.P. Rand, Effects of cholesterol on the structural transitions induced by diacylglycerol in phosphatidylcholine and phosphatidylethanolamine bilayer systems, Biochem. Cell. Biol. 68 (1990) 65-69.

[29] A.J. Kreutzberger, V. Kiessling, L.K. Tamm, High cholesterol obviates a prolonged hemifusion intermediate in fast SNARE-mediated membrane fusion, Biophys. J. 109 (2015) 319-329.

[30] A.J.B. Kreutzberger, V. Kiessling, C. Stroupe, B. Liang, J. Preobraschenski, M. Ganzella, M.A.B. Kreutzberger, R. Nakamoto, R. Jahn, J.D. Castle, L.K. Tamm, In vitro fusion of single synaptic and dense core vesicles reproduces key physiological properties, Nat. Commun. 10 (2019) 3904.

[31] L. Wu, K.C. Courtney, E.R. Chapman, Cholesterol stabilizes recombinant exocytic fusion pores by altering membrane bending rigidity, Biophys. J. 120 (2021) 1367-1377.

[32] J. Zhang, R. Xue, W.Y. Ong, P. Chen, Roles of cholesterol in vesicle fusion and motion, Biophys. J. 97 (2009) 1371-1380.

[33] S. Ge, J.G. White, C.L. Haynes, Critical role of membrane cholesterol in exocytosis revealed by single platelet study, ACS Chem. Biol. 5 (2010) 819-828.

[34] C.R. Wasser, M. Ertunc, X. Liu, E.T. Kavalali, Cholesterol-dependent balance between evoked and spontaneous synaptic vesicle recycling, J. Physiol. 579 (2007) 413-429.

[35] Y. Xu, D.K. Toomre, J.S. Bogan, M. Hao, Excess cholesterol inhibits glucosestimulated fusion pore dynamics in insulin exocytosis, J. Cell. Mol. Med. 21 (2017) 2950-2962.

[36] A.J. Mercer, R.J. Szalewski, S.L. Jackman, M.J. Van Hook, W.B Thoreson, Regulation of presynaptic strength by controlling Ca2 + channel mobility: effects of cholesterol depletion on release at the cone ribbon synapse, J. Neurophysiol. 107 (2012) 3468-3478.

[37] N. Kato, M. Nakanishi, N. Hirashima, Cholesterol depletion inhibits storeoperated calcium currents and exocytotic membrane fusion in RBL-2H3 cells, Biochemistry 42 (2003) 11808-11814.

[38] M. Kreft, M. Stenovec, M. Rupnik, S. Grilc, M. Krzan, M. Potokar, T. Pangrsic, P. G. Haydon, R. Zorec, Properties of $\mathrm{Ca}(2+)$-dependent exocytosis in cultured astrocytes, Glia 46 (2004) 437-445.

[39] E. Lasic, M. Lisjak, A. Horvat, M. Bozic, A. Sakanovic, G. Anderluh, A. Verkhratsky, N. Vardjan, J. Jorgacevski, M. Stenovec, R. Zorec, Astrocyte Specific Remodeling of Plasmalemmal Cholesterol Composition by Ketamine Indicates a New Mechanism of Antidepressant Action, Sci. Rep. 9 (2019) 10957.

[40] L.J. Breckenridge, W. Almers, Currents through the fusion pore that forms during exocytosis of a secretory vesicle, Nature 328 (1987) 814-817.

[41] K. Lollike, N. Borregaard, M. Lindau, The exocytotic fusion pore of small granules has a conductance similar to an ion channel, J. Cell Biol. 129 (1995) 99-104.

[42] J.R. Coorssen, H. Schmitt, W. Almers, Ca2 + triggers massive exocytosis in Chinese hamster ovary cells, EMBO J. 15 (1996) 3787-3791.

[43] B. Rituper, A. Gucek, J. Jorgacevski, A. Flasker, M. Kreft, R. Zorec, Highresolution membrane capacitance measurements for the study of exocytosis and endocytosis, Nat. Protoc. 8 (2013) 1169-1183.
[44] J.P. Schwartz, D.J. Wilson, Preparation and characterization of type 1 astrocytes cultured from adult rat cortex, cerebellum, and striatum, Glia 5 (1992) 75-80.

[45] S.K. Loftus, J.A. Morris, E.D. Carstea, J.Z. Gu, C. Cummings, A. Brown, J. Ellison, K. Ohno, M.A. Rosenfeld, D.A. Tagle, P.G. Pentchev, W.J. Pavan, Murine model of Niemann-Pick C disease: mutation in a cholesterol homeostasis gene, Science 277 (1997) 232-235.

[46] M. Khan, S. Gasser, Generating primary fibroblast cultures from mouse ear and tail tissues, J. Vis. Exp. (2016).

[47] K. Lollike, M. Lindau, Membrane capacitance techniques to monitor granule exocytosis in neutrophils, J. Immunol. Methods 232 (1999) 111-120.

[48] A.E. Spruce, L.J. Breckenridge, A.K. Lee, W. Almers, Properties of the fusion pore that forms during exocytosis of a mast cell secretory vesicle, Neuron 4 (1990) 643-654.

[49] M.A. Churchward, T. Rogasevskaia, J. Hofgen, J. Bau, J.R. Coorssen, Cholesterol facilitates the native mechanism of Ca2+-triggered membrane fusion, J. Cell. Sci. 118 (2005) 4833-4848.

[50] J.N. Kapur, P.K. Sahoo, A.K.C. Wong, A New method for gray-level picture thresholding using the entropy of the histogram, Comput. Gr. Image Process. 29 (1985) 273-285.

[51] W.-.H. Tsai, Moment-preserving thresholding: a New Approach, IEEE Computer Society Press, 1995, pp. 44-60. In: Document image analysis (eds. OG Lawrence, K Rangachar).

[52] S. Bolte, F.P. Cordelieres, A guided tour into subcellular colocalization analysis in light microscopy, J. Microsc. -Oxford 224 (2006) 213-232.

[53] T.W. Ridler, S. Calvard, Picture thresholding using an iterative selection method, IEEE Trans. Syst. Man Cybern. 8 (1978) 630-632.

[54] M. Holtta-Vuori, R.L. Uronen, J. Repakova, E. Salonen, I. Vattulainen, P. Panula, Z. Li, R. Bittman, E. Ikonen, BODIPY-cholesterol: a new tool to visualize sterol trafficking in living cells and organisms, Traffic 9 (2008) 1839-1849.

[55] J.S. Bogan, Y. Xu, M. Hao, Cholesterol accumulation increases insulin granule size and impairs membrane trafficking, Traffic 13 (2012) 1466-1480.

[56] M. Maekawa, Domain 4 (D4) of perfringolysin O to visualize cholesterol in cellular membranes-the update, Sensors (2017) 17 (Basel.

[57] Y. Ohno-Iwashita, M. Iwamoto, S. Ando, K. Mitsui, S. Iwashita, A modified thetatoxin produced by limited proteolysis and methylation: a probe for the functional study of membrane cholesterol, Biochim. Biophys. Acta 1023 (1990) 441-448.

[58] M. Stenovec, M. Kreft, I. Poberaj, W.J. Betz, R. Zorec, Slow spontaneous secretion from single large dense-core vesicles monitored in neuroendocrine cells, FASEB J. 18 (2004) 1270-1272.

[59] P.S. Dannies, Prolactin and growth hormone aggregates in secretory granules: the need to understand the structure of the aggregate, Endocr. Rev. 33 (2012) 254-270.

[60] M. Stenovec, P.P. Goncalves, R. Zorec, Peptide hormone release monitored from single vesicles in "membrane lawns" of differentiated male pituitary cells: sNAREs and fusion pore widening, Endocrinology 154 (2013) 1235-1246.

[61] A.J. Cochilla, J.K. Angleson, W.J. Betz, Monitoring secretory membrane with FM1-43 fluorescence, Annu. Rev. Neurosci. 22 (1999) 1-10.

[62] M. Stenovec, S. Trkov, M. Kreft, R. Zorec, Alterations of calcium homoeostasis in cultured rat astrocytes evoked by bioactive sphingolipids, Acta Physiol. (Oxf) 212 (2014) 49-61.

[63] M. Stenovec, M. Kreft, S. Grilc, T. Pangrsic, R. Zorec, EAAT2 density at the astrocyte plasma membrane and $\mathrm{Ca}(2+)$-regulated exocytosis, Mol. Membr. Biol. 25 (2008) 203-215.

[64] V. Gundersen, J. Storm-Mathisen, L.H. Bergersen, Neuroglial transmission, Physiol. Rev. 95 (2015) 695-726.

[65] N. Vardjan, M. Stenovec, J. Jorgacevski, M. Kreft, R. Zorec, Subnanometer fusion pores in spontaneous exocytosis of peptidergic vesicles, J. Neurosci. 27 (2007) $4737-4746$.

[66] A. Gucek, J. Jorgacevski, P. Singh, C. Geisler, M. Lisjak, N. Vardjan, M. Kreft, A. Egner, R. Zorec, Dominant negative SNARE peptides stabilize the fusion pore in a narrow, release-unproductive state, Cell. Mol. Life Sci. 73 (2016) 3719-3731.

[67] K.G. Ormerod, T.P. Rogasevskaia, J.R. Coorssen, A.J. Mercier, Cholesterolindependent effects of methyl-beta-cyclodextrin on chemical synapses, PLoS ONE 7 (2012) e36395.

[68] R.J. Bruckner, S.S. Mansy, A. Ricardo, L. Mahadevan, J.W Szostak, Flip-flopinduced relaxation of bending energy: implications for membrane remodeling, Biophys. J. 97 (2009) 3113-3122.

[69] L. Liscum, Niemann-Pick type C mutations cause lipid traffic jam, Traffic 1 (2000) 218-225.

[70] W. Helfrich, Elastic properties of lipid bilayers: theory and possible experiments, Z Naturforsch C 28 (1973) 693-703.

[71] S.O. Yesylevskyy, T. Rivel, C. Ramseyer, The influence of curvature on the properties of the plasma membrane. Insights from atomistic molecular dynamics simulations, Sci. Rep. 7 (2017) 16078.

[72] Z. Chen, R.P. Rand, The influence of cholesterol on phospholipid membrane curvature and bending elasticity, Biophys. J. 73 (1997) 267-276.

[73] S. Mukherjee, F.R. Maxfield, Lipid and cholesterol trafficking in NPC, Biochim. Biophys. Acta 1685 (2004) 28-37.

[74] M.E. Higgins, J.P. Davies, F.W. Chen, Y.A. Ioannou, Niemann-Pick C1 is a late endosome-resident protein that transiently associates with lysosomes and the trans-Golgi network, Mol. Genet. Metab. 68 (1999) 1-13.

[75] M. Kreft, J. Jorgacevski, M. Stenovec, R. Zorec, Angstrom-size exocytotic fusion pore: implications for pituitary hormone secretion, Mol. Cell. Endocrinol. 463 (2018) 65-71. 
[76] H.C. Chiang, W. Shin, W.D. Zhao, E. Hamid, J. Sheng, M. Baydyuk, P.J. Wen, A. Jin, F. Momboisse, L.G Wu, Post-fusion structural changes and their roles in exocytosis and endocytosis of dense-core vesicles, Nat. Commun. 5 (2014) 3356

[77] P.J. Wen, S. Grenklo, G. Arpino, X. Tan, H.S. Liao, J. Heureaux, S.Y. Peng, H. C. Chiang, E. Hamid, W.D. Zhao, W. Shin, T. Nareoja, E. Evergren, Y. Jin, R. Karlsson, S.N. Ebert, A. Jin, A.P. Liu, O. Shupliakov, L.G Wu, Actin dynamics provides membrane tension to merge fusing vesicles into the plasma membrane, Nat. Commun. 7 (2016) 12604.

[78] W. Shin, G. Arpino, S. Thiyagarajan, R. Su, L. Ge, Z. McDargh, X. Guo, L. Wei, O. Shupliakov, A. Jin, B. O'Shaughnessy, L.G Wu, Vesicle Shrinking and Enlargement Play Opposing Roles in the Release of Exocytotic Contents, Cell Rep. 30 (2020) 421-431, e7.

[79] Q. Zhang, B. Liu, Y. Li, L. Yin, M. Younus, X. Jiang, Z. Lin, X. Sun, R. Huang, B. Liu, Q. Wu, F. Zhu, Z. Zhou, Regulating quantal size of neurotransmitter release through a GPCR voltage sensor, Proc. Natl. Acad. Sci. U. S. A. 117 (2020) 26985-26995.

[80] M. Mahadeo, K.L. Furber, S. Lam, J.R. Coorssen, E.J. Prenner, Secretory vesicle cholesterol: correlating lipid domain organization and Ca2+ triggered fusion, Biochim. Biophys. Acta 1848 (2015) 1165-1174.

[81] R.H. Chow, L. von Ruden, E. Neher, Delay in vesicle fusion revealed by electrochemical monitoring of single secretory events in adrenal chromaffin cells, Nature 356 (1992) 60-63.

[82] T. Pangrsic, M. Potokar, P.G. Haydon, R. Zorec, M. Kreft, Astrocyte swelling leads to membrane unfolding, not membrane insertion, J. Neurochem. 99 (2006) 514-523.

[83] S. Ozawa, O. Sand, Action potentials in non-tumor cells from the anterior pituitary gland, Experientia 34 (1978) 542-544.

[84] S.N. Akerman, R. Zorec, T.R. Cheek, R.B. Moreton, M.J. Berridge, W.T. Mason, Fura-2 imaging of thyrotropin-releasing hormone and dopamine effects on calcium homeostasis of bovine lactotrophs, Endocrinology 129 (1991) 475-488.

[85] R. Zorec, A. Araque, G. Carmignoto, P.G. Haydon, A. Verkhratsky, V. Parpura, Astroglial excitability and gliotransmission: an appraisal of $\mathrm{Ca} 2+$ as a signalling route, ASN Neuro (2012) 4

[86] A. Verkhratsky, V. Parpura, N. Vardjan, R. Zorec, Physiology of Astroglia, Adv. Exp. Med. Biol. 1175 (2019) 45-91.

[87] N. Vardjan, R. Zorec, Excitable Astrocytes: ca(2+)- and cAMP-Regulated Exocytosis, Neurochem. Res. 40 (2015) 2414, 14.

[88] N. Vardjan, V. Parpura, R. Zorec, Loose excitation-secretion coupling in astrocytes, Glia 64 (2016) 655-667.

[89] A. Verkhratsky, M. Matteoli, V. Parpura, J.P. Mothet, R. Zorec, Astrocytes as secretory cells of the central nervous system: idiosyncrasies of vesicular secretion, EMBO J. (2016).

[90] V. Parpura, R. Zorec, Gliotransmission: exocytotic release from astrocytes, Brain Res. Rev. 63 (2010) 83-92.
[91] P.J. Magistretti, I. Allaman, Lactate in the brain: from metabolic end-product to signalling molecule, Nat. Rev. Neurosci. 19 (2018) 235-249.

[92] M.E. Gibbs, D.G. Anderson, L. Hertz, Inhibition of glycogenolysis in astrocytes interrupts memory consolidation in young chickens, Glia 54 (2006) 214-222.

[93] D.H. Mauch, K. Nägler, S. Schumacher, C. Göritz, E.C. Müller, A. Otto, F. W. Pfrieger, CNS synaptogenesis promoted by glia-derived cholesterol, Science 294 (2001) 1354-1357.

[94] M.A. Churchward, T. Rogasevskaia, D.M. Brandman, H. Khosravani, P. Nava, J. K. Atkinson, J.R. Coorssen, Specific lipids supply critical negative spontaneous curvature-an essential component of native Ca2+-triggered membrane fusion, Biophys. J. 94 (2008) 3976-3986.

[95] P.B. Canham, The minimum energy of bending as a possible explanation of the biconcave shape of the human red blood cell, J. Theor. Biol. 26 (1970) 61-81.

[96] E.M. Blokhuis, M. D'Agostino, A. Mayer, H.J Risselada, Fusion Pores Live on the Edge, J. Phys. Chem. Lett. 11 (2020) 1204-1208.

[97] W. Rawicz, K.C. Olbrich, T. McIntosh, D. Needham, E. Evans, Effect of chain length and unsaturation on elasticity of lipid bilayers, Biophys. J. 79 (2000) 328-339.

[98] J. Jorgacevski, M. Potokar, S. Grilc, M. Kreft, W. Liu, J.W. Barclay, J. Buckers, R. Medda, S.W. Hell, V. Parpura, R.D. Burgoyne, R. Zorec, Munc18-1 tuning of vesicle merger and fusion pore properties, J. Neurosci. 31 (2011) 9055-9066.

[99] M.B.L. Winkler, R.T. Kidmose, M. Szomek, K. Thaysen, S. Rawson, S.P. Muench, D. Wustner, B.P. Pedersen, Structural Insight into Eukaryotic Sterol Transport through Niemann-Pick Type C Proteins, Cell 179 (2019) 485-497, e18.

[100] D. Hoglinger, T. Burgoyne, E. Sanchez-Heras, P. Hartwig, A. Colaco, J. Newton, C. E. Futter, S. Spiegel, F.M. Platt, E.R. Eden, NPC1 regulates ER contacts with endocytic organelles to mediate cholesterol egress, Nat. Commun. 10 (2019) 4276.

[101] H.J. Deuling, W. Helfrich, Red blood cell shapes as explained on the basis of curvature elasticity, Biophys. J. 16 (1976) 861-868.

[102] U. Seifert, K. Berndl, R. Lipowsky, Shape transformations of vesicles: phase diagram for spontaneous- curvature and bilayer-coupling models, Phys. Rev. A 44 (1991) 1182-1202.

[103] S. Svetina, B. Zeks, Membrane bending energy and shape determination of phospholipid vesicles and red blood cells, Eur. Biophys. J. 17 (1989) 101-111.

[104] F. Julicher, U. Seifert, Shape equations for axisymmetric vesicles: a clarification, Phys. Rev. E Stat. Phys. Plasmas Fluids Relat. Interdiscip. Topics 49 (1994) 4728-4731.

[105] P.W. Kuchel, B.G. Ralston, Theory and Problems of Biochemistry. Schaum's Outline/Mcgraw-Hill, 1988. New York.

[106] J.E. Heuser, Review of electron microscopic evidence favouring vesicle exocytosis as the structural basis for quantal release during synaptic transmission, Q. J. Exp. Physiol. 74 (1989) 1051-1069. 